\title{
Gravitational dynamics in a $2+1+1$ decomposed spacetime along nonorthogonal double foliations: Hamiltonian evolution and gauge fixing
}

\author{
Cecília Gergely, Zoltán Keresztes, and László Á. Gergely \\ Institute of Physics, University of Szeged, Dóm tér 9, 6r720 Szeged, Hungary
}

(Dated: May 2, 2019)

\begin{abstract}
Motivated by situations with temporal evolution and spatial symmetries both singled out, we develop a new $2+1+1$ decomposition of spacetime, based on a nonorthogonal double foliation. Time evolution proceeds along the leaves of the spatial foliation. We identify the gravitational variables in the velocity phase-space as the 2-metric (induced on the intersection $\Sigma_{t \chi}$ of the hypersurfaces of the foliations), the $2+1$ components of the spatial shift vector, together with the extrinsic curvature, normal fundamental form and normal fundamental scalar of $\Sigma_{t \chi}$, all constructed with the normal to the temporal foliation. This work generalizes a previous decomposition based on orthogonal foliations, a formalism lacking one metric variable, now reintroduced. The new metric variable is related to (i) the angle of a Lorentz-rotation between the nonorthogonal bases adapted to the foliations, and (ii) to the vorticity of these basis vectors. As a first application of the formalism, we work out the Hamiltonian dynamics of general relativity in terms of the variables identified as canonical, generalizing previous work. As a second application we present the unambiguous gaugefixing suitable to discuss the even sector scalar-type perturbations of spherically symmetric and static spacetimes in generic scalar-tensor gravitational theories, which has been obstructed in the formalism of orthogonal double foliation.
\end{abstract}

\section{INTRODUCTION}

The modern theory of gravitation, general relativity (GR) has been successfully tested multiple times on the Solar Sytem scale. When confronted with observations on both galactic scales and beyond, agreement with predictions can however be reached only at the price of introducing dark matter and dark energy, neither of them identified or detected by other means than gravitational. Lacking indications on manifestations of these forms of matter in the Standard Model interactions, they could be included in the gravitational sector, either as geometric modifications arising from possible higher-order dynamics or as an excess of fields representing gravity beyond the metric tensor, possibly including scalars, vectors, 2form fields or even a second metric. As a rule, the physical metric couples to these in a nonminimal way, opposed to dark matter/dark energy models, which are coupled minimally. The simplest such model, of a single scalar field complementing the metric has been studied extensively, both from the desire to explain dark matter / dark energy or in order to study inflation.

The most generic single scalar-tensor model described by second order differential equations (hence avoiding Ostrogradski instabilities) for both the metric and the scalar field has been proposed by Horndeski [1] and rediscovered in a modern context in connection with generalized galileons [2].

While allowing for higher order than two, certain beyond Horndeski models could guarantee that the propagating degrees of freedom (d.o.f.) still evolve according to a second order dynamics. Indeed, an effective field theory (EFT) of cosmological perturbations has been worked out by Gleyzes et al. [3, 4], based on (a) a Lagrangian depending on the lapse function and some geometrical scalar quantities emerging in the Arnowitt-Deser-Misner
(ADM) decomposition on the flat Friedmann-LemaîtreRobertson-Walker (FLRW) background and (b) the unitary gauge, allowing to absorb the scalar field perturbation by an adequate time coordinate choice (the lapse is then associated with the corresponding constant scalarfield hypersurfaces). The linear perturbation equations contain time derivatives at second order, although spatial derivatives could be of higher order (in the Horndeski subclass the latter are also of second order). A generalization for two scalar fields representing dark matter and dark energy has been advanced in Ref. 5]. Another generalization has been discussed in Ref. [6], referring to perturbations of a spherically symmetric and static background, treated similarly.

These theories should obey the requirements of

(A) stability, guaranteed by the avoidance of both scalar ghosts (no negative kinetic term in the second order Lagrangian governing the evolution of linear perturbations) and Laplacian instabilities (no negative sound speed squared),

(B) agreement with Solar System tests, notably the Vainshtein mechanism suppressing the propagation of the fifth force inside the Solar System (no $L_{5}$ contribution to the Horndeski Lagrangian) [7-9],

(C) agreement with weak lensing observations (constraints from deviations from the Newtonian law and light bending by simultaneous fitting of x-ray and lensing profiles of galaxy clusters) [10].

The recent detections of gravitational waves from 10 coalescing binary black holes and one neutron star merger by the LIGO Scientific Collaboration and Virgo Collaboration [11 17] have added new constraints. On the one hand, the mass of the graviton has been severely constrained by testing a massive dispersion relation [18]. 
Then a wide family of dispersion relations [19] were tested, disruling [13] Lorentz-violation, Hořava-Lifsic theories, certain extra dimensional, multifractal theories, doubly special relativity and setting an even harder constraint on the graviton mass at $5.0 \times 10^{-23} \mathrm{eV} / \mathrm{c}^{2}[20]$. On the other hand the small difference in the arrival time of the gravitational waves from a neutron star coalescence [16] and accompanying $\gamma$-radiation confirmed that the tensorial gravitational modes propagate with the speed of light within $-3 \times 10^{-15}$ and $+7 \times 10^{-16}$ accuracy [21]. By exploring previously existing analyses on the Laplacian stability and ghost avoidance in Horndeski theories [22, 23], from these constraints the $L_{5}$ contribution has been disruled once again, together with the kinetic term dependence of $L_{4}$ 24 26]. A slightly less restrictive condition emerged for the beyond Horndeski models. Further, three of the five parameters appearing in the effective theory of dark energy were severely constrained by combining the gravity wave results with galaxy cluster observations [27].

The stability of spherically symmetric, static spacetimes has been discussed for both the odd [28] and even modes [29] of the perturbations in Horndeski theories, also for the odd modes in the beyond Horndeski theories [6]. The latter relied on a double foliation of spacetime along orthogonal spatial and temporal leaves, developed in Refs. [30, 31]. Three independent background dynamical equations were identified and the conditions for avoidance of ghosts and Laplacian instabilities of the odd mode perturbations established.

The formalism of the orthogonal double foliation relies on the extensive use of adapted metric variables, which bear the role of canonical coordinates and on embedding variables (extrinsic curvatures, normal fundamental forms and normal fundamental scalars of the 2-surfaces generated by the intersection of the foliations), some of them emerging as canonical momenta, others as pure spatial derivatives of the coordinates. The odd sector of perturbations of spherically symmetric, static spacetimes has been analyzed in terms of these quantities [6].

Spacetime perturbations can also be discussed through other decomposition techniques, including: (I) the first order system of 70 coupled differential equations for 50 independent variables of the black hole perturbation formalism à la Chandrasekhar [32], based on the NewmanPenrose formalism (an $1+1+1+1$ decomposition); (II) the formalism based on the numerous variables arising from a $2+1+1$ decomposition based on kinematical quantities (optical scalars), supplemented by the electric and magnetic projection of the Weyl tensor [33, 34]; (III) a $(2+1)+1$ decomposition based on the introduction of the quotient space defined by the orbits of a rotational Killing vector [35, 36]; (IV) a temporal foliation followed by a further $2+1$ slicing to deal with axisymmetric and stationary configurations [37], generalized later on for a $2+1$ foliation of a hypersurface with arbitrary causal character [38, 39], a technique also employed in Ref. [40] for identifying a hyperbolic system in the constraint struc- ture, rewritten in terms of the $2+1$ decomposition of the extrinsic curvature of the hypersurfaces explored previously in the orthogonal double foliation formalism of Ref. [30]; (V) the standard metric perturbation formalism, explored in a spherically symmetric, static setup in Refs. [28, 29]. The advantage of the orthogonal double foliation formalism over the first two consist in its substantially reduced number of variables. A comparison with the third and fourth has been presented in [30]. The third relies heavily on the use of a Killing vector, which is not a necessity for the orthogonal double foliation. Although the fourth approach contains the same number of metric variables (9), it does not employ all geometric quantities playing an essential role in Refs. 30, 31]. In particular, Ref. 38 introduces a second fundamental form combining a set of dynamical and nondynamical variables explored in Refs. [30, 31], a normal fundamental form but no normal fundamental scalar. Finally, the advantage over the metric perturbation formalism is the canonical (geometrodynamical) interpretation of the variables. ${ }^{1}$

The simplicity of the orthogonal double foliation of Refs. 30, 31] however required to waste one gauge d.o.f. for imposing the orthogonality requirement after the perturbation. This hampered the discussion of the even modes, carrying an arbitrary function of time, hence losing their physical interpretation [6]

It is the purpose of the present paper to lift the condition of orthogonality of the two foliations in order to recover the full power of gauge fixing and open the way for the discussion of the even mode perturbations in generic scalar-tensor theories on a spherically symmetric, static background, complementing the similar discussion of the odd sector.

The paper is organized as follows. In Sec. II we develop the new $2+1+1$ decomposition of the spacetime $\mathcal{B}$ based on two nonorthogonal foliations, one of them temporal $\left(\mathcal{S}_{t}\right.$, characterized by constant $\left.t\right)$, the other one spatial $\left(\mathfrak{M}_{\chi}\right.$, with constant $\left.\chi\right)$. This generalizes the formalism of the orthogonal double foliation of spacetime, developed in Refs. [30, 31], by allowing for a 10th metric function $\mathcal{N}$. We adapt suitable bases to both foliations, then give the evolutions along the $\partial / \partial t$ and $\partial / \partial \chi$ con-

\footnotetext{
${ }^{1}$ Other spacetime decomposition techniques are also known. Applying the formalism developed in the seminal monograph [41], a $2+2$ breakup of the field equations was advanced in Ref. [42] with the aim of identifying the gravitational d.o.f. in the so-called conformal two-structure (the latter representing the information on how the family of selected 2-surfaces is embedded in a 3surface). For the discussion of the initial value problem Ref. [43] developed the $2+2$ decomposition of spacetime in detail, based on space-like 2 -surfaces $\{S\}$ rigged by a dyad basis given by their two mutually orthogonal normals (and the respective orthogonal 3 -foliations). Then the covariant derivatives of these normals were decomposed in terms of the extrinsic curvatures of $\{S\}$, the induced connection of the timelike 2-surface $\{T\}$ spanned by the dyad basis and the curvature tensor of $\{T\}$. The Einstein equations were decomposed accordingly.
} 
gruences (tangent to $\mathfrak{M}_{\chi}$ and $\mathcal{S}_{t}$, respectively) in both bases. Two of the basis vectors (tangent to the intersection $\Sigma_{t \chi}$ of $\mathcal{S}_{t}$ and $\mathfrak{M}_{\chi}$ ) are common in both bases, while the other two pairs are related by a Lorentz rotation with angle $\phi=\tanh ^{-1}(\mathcal{N} / N)$, where $N$ is the lapse function. Another geometric interpretation of the 10th metric function arises as the vorticity of the basis vectors orthogonal to both the hypersurface normals (of the same basis) and to $\Sigma_{t \chi}$. This is shown here through the discussion of the algebras of each basis vectors and in the discussion of the vorticities in the two Appendices.

In Sec. III we characterize the embedding in terms of extrinsic curvatures, normal fundamental forms and normal fundamental scalars of the hypersurface normals, also introduce the $2+1$ decomposed form of the curvature of their congruences (their nongravitational accelerations). For the basis vectors orthogonal to them we introduce similar quantities. We establish the interconnections among all those geometric quantities. In Sec. IV we also derive their connection with the time- and $\chi$-derivatives of the metric functions. This enables us to select those geometric variables, which bear a dynamical role, e.g., connected to canonical momenta.

As a first application, we present the Hamiltonian formalism of general relativity in the $2+1+1$ decomposed form in Sec. $\nabla$ We derive the canonical momenta, then the Hamiltonian and diffeomorphism constraints and the boundary terms of the action, all in terms of canonical data defined on $\Sigma_{t \chi}$. We recover previous results of Ref. [31] applying for the orthogonal double foliation in the vanishing $\mathcal{N}$ limit. The nonorthogonality of the foliations also generates new terms.

Then, in Sec.VI we explore the diffeomorphism gauge freedom for fixing the perturbations on the static and spherically symmetric background of beyond Horndeski theories in an unambiguous way. This result opens up the possibility for the discussion of the even sector of the perturbations. Although the unambiguous gauge fixing is different from the one employed for the odd sector in Ref. [6], the results of the stability analysis presented there are unaffected, as the two sectors decouple.

In Sec. VII we present our conclusions. Two Appendices are devoted to discuss the consequences of the hypersurface orthogonality of the normal basis vectors and the interpretation of the vorticities of the complementary basis vectors in terms of the geometric quantities introduced in the main body of the paper.

We use the abstract index notation throughout the paper. Latin and greek indices, respectively, denote 4-dimensional spacetime and 3-dimensional spatial abstract indices. Boldface lower- and uppercase indices differentiate among 2-dimensional and 4-dimensional basis vectors, respectively. 4-dimensional quantities will carry a distinguishing tilde sign, while 3-dimensional quantities a overhat (or reversed overhat) sign. Tensors defined both on the full spacetime and on lower-dimensional (hyper)surfaces carry Latin indices, the latter obeying the required projection conditions. Quantities defined on the background in a perturbational setup carry an overbar. Round or square brackets on indices denote symmetrization or antisymmetrization, respectively.

\section{THE NONORTHOGONAL $2+1+1$ DECOMPOSITION OF SPACETIME}

Let $\mathcal{B}$ be a 4 -dimensional manifold with metric $\tilde{g}_{a b}$ of Lorentzian signature. We assume the manifold admits both a timelike and a spacelike foliation. In this section we generalize the formalism of [30, 31] by dropping the orthogonality requirement of the foliations $\mathcal{S}_{t}$ (with constant time coordinate $t$ ) and $\mathfrak{M}_{\chi}$ (with constant space coordinate $\chi$ ).

On the tangent space of the doubly-foliable spacetime $\mathcal{B}$ we introduce the bases $e_{\mathbf{A}}=\left\{\partial / \partial t, \partial / \partial \chi, E_{\mathbf{i}}\right\}$ (with $E_{\mathbf{i}}$ some basis elements of the tangent space of $\left.\Sigma_{t \chi}\right)$ and its dual $e^{\mathbf{B}}=\left\{d t, d \chi, E^{\mathbf{j}}\right\}$ on the respective cotangent space.

Let $n^{a}$ be the (timelike) unit normal to $\mathcal{S}_{t}$, while $m^{a}$ the (spacelike) unit normal to both $n^{a}$ and $\Sigma_{t \chi}$. With them we introduce the basis $f_{\mathbf{A}}=\left\{n, m, F_{\mathbf{i}}\right\}$ adapted to $\mathcal{S}_{t}$ (with $F_{\mathbf{i}}$ basis elements of the tangent space of $\Sigma_{t \chi}$ ) and its dual $f^{\mathbf{B}}=\left\{\bar{n}, \bar{m}, F^{\mathbf{j}}\right\}$. The (spacelike) unit normal to $\mathfrak{M}_{\chi}$ is $l^{a}$, while $k^{a}$ denotes the (timelike) unit normal to both $l^{a}$ and $\Sigma_{t \chi}$. The basis adapted to $\mathfrak{M}_{\chi}$ is $g_{\mathbf{A}}=\left\{k, l, G_{\mathbf{i}}\right\}$ (where $G_{\mathbf{i}}$ are basis elements of the tangent space of $\left.\Sigma_{t \chi}\right)$, with $g^{\mathbf{B}}=\left\{\bar{k}, \bar{l}, G^{\mathbf{j}}\right\}$ its dual.

For simplicity one can chose coordinate basis vectors $E_{\mathbf{i}}=F_{\mathbf{i}}=G_{\mathbf{i}}=\partial / \partial y^{\mathrm{i}}$. From the causal character of the basis vectors and from the duality relations we get

$$
\begin{aligned}
& \bar{n}_{a}=-n_{a}, \quad \bar{m}_{a}=m_{a}, \\
& \bar{k}_{a}=-k_{a}, \quad \bar{l}_{a}=l_{a} .
\end{aligned}
$$

\section{A. The induced metric}

The 4-metric $\tilde{g}_{a b}$ can be decomposed in two equivalent ways

$$
\begin{aligned}
& \tilde{g}_{a b}=-n_{a} n_{b}+m_{a} m_{b}+g_{a b}, \\
& \tilde{g}_{a b}=-k_{a} k_{b}+l_{a} l_{b}+g_{a b} .
\end{aligned}
$$

As usual $\tilde{g}_{a}^{b} \equiv \delta_{a}^{b}$, while the mixed form of the induced metric $g_{a b}$ projects to $\Sigma_{t \chi}$. With this projection, both covariant derivatives and Lie derivatives along a congruence $V^{a}$ of any 4-dimensional tensor $\tilde{T}_{b_{1} \ldots b_{r}}^{a_{1} \ldots a_{r}}$ could be projected onto $\Sigma_{t \chi}$ :

$$
\begin{gathered}
D_{a} \tilde{T}_{b_{1} \ldots b_{q}}^{a_{1} \ldots a_{r}} \equiv g_{a}^{c} g_{c_{1}}^{a_{1}} \ldots g_{c_{r}}^{a_{r}} g_{b_{1}}^{d_{1}} \ldots g_{b_{q}}^{d_{q}} \tilde{\nabla}_{c} \tilde{T}_{d_{1} \ldots d_{q}}^{c_{1} \ldots c_{r}} \\
\mathfrak{L}_{\mathbf{V}} \tilde{T}_{b_{1} \ldots b_{q}}^{a_{1} \ldots a_{r}} \equiv g_{c_{1}}^{a_{1}} \ldots g_{c_{r}}^{a_{r}} g_{b_{1}}^{d_{1}} \ldots g_{b_{q}}^{d_{q}} \tilde{\mathfrak{L}}_{\mathbf{V}} \tilde{T}_{d_{1} \ldots d_{q}}^{c_{1} \ldots c_{r}}
\end{gathered}
$$

We note that whenever $\tilde{T}_{b_{1} \ldots b_{q}}^{a_{1} \ldots a_{r}}$ is a projected object onto $\Sigma_{t \chi}$, the expression $D_{a} \tilde{T}_{b_{1} \ldots b_{q}}^{a_{1} \ldots a_{r}}$ is exactly the covariant derivative in $\Sigma_{t \chi}$ (which annihilates $g_{a b}$ ), while 
$\mathfrak{L}_{\mathbf{V}} \tilde{T}_{b_{1} \ldots b_{q}}^{a_{1} \ldots a_{r}}$ describes an evolution along the congruence $V^{a}$ (it represents the partial derivative with respect to the adapted coordinate $v$, thus $\mathbf{V}=\partial / \partial v$, where $v$ could be either $t$ or $\chi)$. Otherwise they become but notations, as they fail to obey the Leibniz rule [30]).

\section{B. Evolutions in the $f_{\mathbf{A}}$ basis}

The first two elements of the coordinate basis $e_{\mathbf{A}}$, representing evolution vectors can be generically decomposed in the $f_{\mathbf{A}}$ basis as

$$
\begin{aligned}
& \left(\frac{\partial}{\partial t}\right)^{a}=N n^{a}+N^{a}+\mathcal{N} m^{a}, \\
& \left(\frac{\partial}{\partial \chi}\right)^{a}=M m^{a}+M^{a}+\mathcal{M} n^{a} .
\end{aligned}
$$

Here $N^{a}$ and $M^{a}(\mathcal{N}$ and $\mathcal{M})$ are the components of the 3-dimensional shift vectors along (orthogonal to) $\Sigma_{t \chi}$, while $N$ and $M$ represent lapse type functions of the respective evolutions. Together with the 3 independent components of $g_{a b}$ there seem to be 11 gravitational variables at this stage, but their number will be reduced to 10. Indeed, the duality relation $\langle d t, \partial / \partial \chi\rangle=0$ implies $\mathcal{M}=0$, which in turn implies through Eq. (6) that $\partial / \partial \chi$ is tangent to $\mathcal{S}_{t}$, see also Fig. 1,

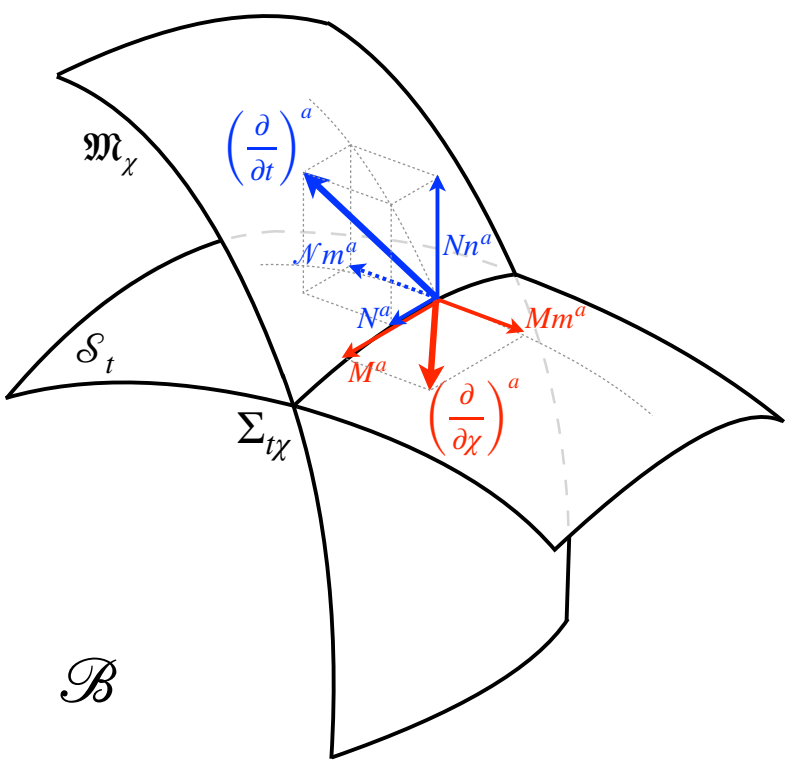

FIG. 1: The decomposition of the temporal and radial evolution vectors in the $f_{\mathbf{A}}$ basis. (For visualization purposes a negative $\mathcal{N}$ was chosen.)

From the rest of the duality relations $\left\langle e^{\mathbf{B}}, e_{\mathbf{A}}\right\rangle=\delta_{\mathbf{A}}^{\mathbf{B}}$ one gets

$$
\begin{aligned}
\bar{n} & =N d t \\
\bar{m} & =\mathcal{N} d t+M d \chi \\
F^{\mathbf{j}} & =N^{\mathbf{j}} d t+M^{\mathbf{j}} d \chi+E^{\mathbf{j}}
\end{aligned}
$$

As $\partial / \partial t$ is timelike and $N^{a}$ spacelike, the inequalities

$$
N^{2}-\mathcal{N}^{2}>g_{a b} N^{a} N^{b} \geq 0
$$

hold, while $\partial / \partial t$ lying in the future light cone implies $N>0$.

We conclude this subsection by giving in Table 1 the algebra of the basis vectors $f_{\mathbf{A}}$. As expected from the Frobenius theorem, the basis vectors $\left\{m, F_{\mathbf{i}}\right\}$ span the tangent space of $\mathcal{S}_{t}$, while from the dual form of the Frobenius theorem the fourth basis vector $n^{a}$ turns out vorticity-free (also shown explicitly in Appendix $\mathrm{A}$ ). The same type of reasoning yields that $m^{a}$ has vorticity (as the component along $m^{a}$ of the $\left[n, F_{\mathbf{j}}\right]$ bracket is nonvanishing, hence the vectors $\left\{n, F_{\mathbf{i}}\right\}$ do not span a hypersurface). This vorticity is given in Appendix $B$ and disappears together with $\mathcal{N}$ in the orthogonal foliation limit employed in Refs. [30, 31]. Hence the vorticity of the basis vector $m^{a}$ is generated by the nonorthogonality of the two foliations.

\section{The role of the 10 th metric variable}

The new element in the formalism as compared with that of Refs. [30, 31] is the shift component $\mathcal{N}$, which reestablishes the number of gravitational variables as 10 , equivalent to the 4-metric variables.

Straightforward calculations employing also the rest of the duality relations $\left\langle f^{\mathbf{B}}, f_{\mathbf{A}}\right\rangle=\delta_{\mathbf{A}}^{\mathbf{B}}=\left\langle g^{\mathbf{B}}, g_{\mathbf{A}}\right\rangle$ and Eqs. (17) lead to the relation between the two adapted bases

$$
\left(\begin{array}{l}
\bar{k} \\
\bar{l}
\end{array}\right)=\left(\begin{array}{cc}
\mathfrak{c} & -\mathfrak{s} \\
-\mathfrak{s} & \mathfrak{c}
\end{array}\right)\left(\begin{array}{l}
\bar{n} \\
\bar{m}
\end{array}\right),
$$

$($ where $\mathfrak{s}=\sinh \phi, \mathfrak{c}=\cosh \phi)$ and

$$
\left(\begin{array}{l}
k^{a} \\
l^{a}
\end{array}\right)=\left(\begin{array}{cc}
\mathfrak{c} & \mathfrak{s} \\
\mathfrak{s} & \mathfrak{c}
\end{array}\right)\left(\begin{array}{c}
n^{a} \\
m^{a}
\end{array}\right),
$$

thus in the form of a Lorentz-rotation. Its angle is defined by

$$
\mathcal{N}=N \tanh \phi
$$

This represents the second geometric interpretation of the 10 th metric variable (beyond the vorticity of $m$ ).

\section{Evolutions in the $g_{\mathrm{A}}$ basis}

With the Lorentz rotations given in the previous subsection it is easy to express the evolution vectors in the $g_{\mathrm{A}}$ basis:

$$
\begin{aligned}
& \left(\frac{\partial}{\partial t}\right)^{a}=\frac{N}{\mathfrak{c}} k^{a}+N^{a} \\
& \left(\frac{\partial}{\partial \chi}\right)^{a}=M\left(-\mathfrak{s} k^{a}+\mathfrak{c} l^{a}\right)+M^{a} .
\end{aligned}
$$




\begin{tabular}{c||c|c|c} 
& {$[n, m]^{a}$} & {$\left[n, F_{\mathbf{j}}\right]^{a}$} & {$\left[m, F_{\mathbf{j}}\right]^{a}$} \\
\hline \hline$n^{a}$ & $\frac{1}{M}\left[\partial_{\chi}(\ln N)-\frac{1}{M} M^{\mathbf{j}} \partial_{\mathbf{j}}(\ln N)\right]$ & $\partial_{\mathbf{j}}(\ln N)$ & 0 \\
$m^{a}$ & $\frac{1}{M N}\left[-\partial_{t} M+\partial_{\chi} \mathcal{N}+N^{\mathbf{j}} \partial_{\mathbf{j}} M-M^{\mathbf{j}} \partial_{\mathbf{j}} \mathcal{N}\right]$ & $\frac{M}{N} \partial_{\mathbf{j}}\left(\frac{\mathcal{N}}{M}\right)$ & $\partial_{\mathbf{j}}(\ln M)$ \\
$F_{\mathbf{i}}^{a}$ & $\frac{1}{M N}\left(-\partial_{t} M^{\mathbf{i}}+\partial_{\chi} N^{\mathbf{i}}+N^{\mathbf{j}} \partial_{\mathbf{j}} M^{\mathbf{i}}-M^{\mathbf{j}} \partial_{\mathbf{j}} N^{\mathbf{i}}\right)$ & $\frac{1}{N}\left[\partial_{\mathbf{j}} N^{\mathbf{i}}-\frac{\mathcal{N}}{M} \partial_{\mathbf{j}} M^{\mathbf{i}}\right]$ & $\frac{\partial_{\mathbf{j}} M^{\mathbf{i}}}{M}$
\end{tabular}

TABLE I: The algebra of the basis vectors $f_{\mathbf{A}}$. The components of the brackets enlisted in the first line along the vectors in the first column are given.

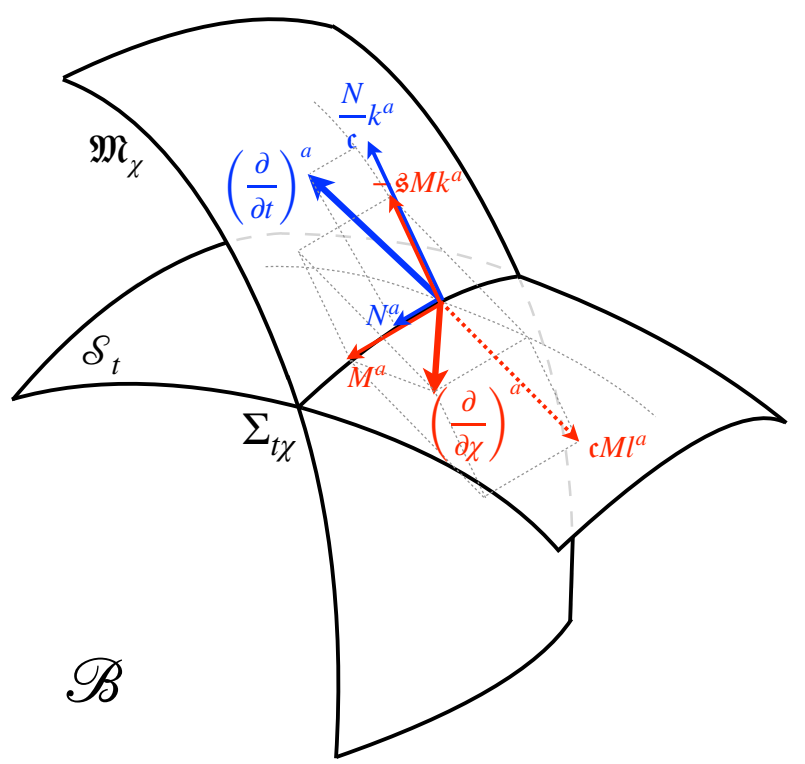

FIG. 2: The decomposition of the temporal and radial evolution vectors in the $g_{\mathbf{A}}$ basis. (For visualization purposes a negative $\mathfrak{s}$ was chosen.)

Remarkably, the evolution vector $\partial / \partial t$ has no component along $l$, hence it is tangent to $\mathfrak{M}_{\chi}$, see also Fig. 2 ,

Further exploring the duality relations one gets

$$
\begin{aligned}
\bar{k} & =-M \mathfrak{s} d \chi+\frac{N}{\mathfrak{c}} d t, \\
\bar{l} & =M \mathfrak{c} d \chi, \\
G^{\mathbf{j}} & =N^{\mathbf{j}} d t+M^{\mathbf{j}} d \chi+E^{\mathbf{j}}=F^{\mathbf{j}} .
\end{aligned}
$$

The algebra of the basis vectors $g_{\mathbf{A}}$ is presented in Table III Again, from the Frobenius theorem, the basis vectors $\left\{k, G_{\mathbf{i}}\right\}$ span the tangent space of $\mathfrak{M}_{\chi}$ and from its dual form the fourth basis vector $l^{a}$ turns out vorticityfree. The vector $k^{a}$ however has vorticity (as the component along $k^{a}$ of the $\left[l, G_{\mathbf{j}}\right]$ bracket is nonvanishing). This vorticity is given in Appendix $\mathrm{B}$ and again disappears with $\mathcal{N}$ in the orthogonal foliation limit employed in Refs. [30, 31]. Hence the vorticity of the basis vector $k^{a}$ is also generated by the nonorthogonality of the two foliations. Finally we note that in the orthogonal foliation limit $\mathcal{N} \rightarrow 0$ the algebras given in Tables IIII coincide and the vorticities of the basis vectors disappear.

\section{CODIMENSION-2 EMBEDDING OF $\Sigma_{t \chi}$}

In this section we introduce a series of geometrical quantities characterizing the embedding of $\Sigma_{t \chi}$ and we analyze their relationship with various coordinate derivatives of the metric variables.

We have defined a total of four normals to the surface $\Sigma_{t \chi}$, two pairs taken from the bases $f_{\mathbf{A}}$ and $g_{\mathbf{A}}$, respectively. With each of them we define an extrinsic curvature, as follows:

$$
\begin{aligned}
K_{a b} & \equiv D_{a} n_{b}=\frac{1}{2} \mathfrak{L}_{\mathbf{n}} g_{a b}, \\
L_{a b} & \equiv D_{a} l_{b}=\frac{1}{2} \mathfrak{L}_{\mathbf{l}} g_{a b}, \\
K_{a b}^{*} & \equiv D_{a} k_{b}=\frac{1}{2} \mathfrak{L}_{\mathbf{k}} g_{a b}, \\
L_{a b}^{*} & \equiv D_{a} m_{b}=\frac{1}{2} \mathfrak{L}_{\mathbf{m}} g_{a b} .
\end{aligned}
$$

All these tensors are symmetric, as shown in the Appendices $\mathrm{A}$ and $\mathrm{B}$.

With the two normals to the hypersurfaces we define the normal fundamental forms of $\Sigma_{t \chi}$ as follows:

$$
\begin{aligned}
\mathcal{K}_{a} & \equiv g_{a}^{c} m^{d} \tilde{\nabla}_{c} n_{d}=g_{a}^{c} m^{d} \tilde{\nabla}_{d} n_{c}, \\
\mathcal{L}_{a} & \equiv-g_{a}^{c} k^{d} \tilde{\nabla}_{c} l_{d}=-g_{a}^{c} k^{d} \tilde{\nabla}_{d} l_{c} .
\end{aligned}
$$

Their second expressions arise from the hypersurfaceorthogonality of the basis vectors $n^{a}$ and $l^{a}$, as proven in Appendix A. It is easy to prove that they are related as

$$
\mathcal{L}_{a}=\mathcal{K}_{a}+D_{a} \phi
$$

By contrast, for the vectors $k^{a}$ and $m^{a}$ (which have vorticity) the similarly defined quantities

$$
\begin{aligned}
\mathcal{K}_{a}^{*} & \equiv g_{a}^{d} l^{c} \tilde{\nabla}_{c} k_{d}, \\
\mathcal{L}_{a}^{*} & \equiv-g_{a}^{d} n^{c} \tilde{\nabla}_{c} m_{d}
\end{aligned}
$$

do not share this interchangeability property. Similarly, one can prove

$$
\mathcal{L}_{a}^{*}=\mathcal{K}_{a}^{*}+D_{a} \phi
$$

The differences $\mathcal{K}_{a}^{*}-\mathcal{L}_{a}$ and $\mathcal{L}_{a}^{*}-\mathcal{K}_{a}$ give the nonvanishing components of the vorticities of $k^{a}$ and $m^{a}$, respectively, as demonstrated in Appendix B 


\begin{tabular}{c||c|c|c} 
& {$[k, l]^{a}$} & {$\left[k, G_{\mathbf{j}}\right]^{a}$} & {$\left[l, G_{\mathbf{j}}\right]^{a}$} \\
\hline \hline$k^{a}$ & $\left\{\partial_{t}\left(\frac{\mathfrak{s}}{N}\right)-N^{\mathbf{j}} \partial_{\mathbf{j}}\left(\frac{\mathfrak{s}}{N}\right)+\frac{\mathfrak{s}}{N}\left[\partial_{t} \ln (M N)-N^{\mathbf{j}} \partial_{\mathbf{j}} \ln (M N)\right]\right.$ & $\partial_{\mathbf{j}}\left(\ln \frac{N}{\mathfrak{c}}\right)$ & $-\frac{N}{\mathfrak{c}^{2} M} \partial_{\mathbf{j}}\left(\frac{\mathfrak{s} c M}{N}\right)$ \\
& $\left.+\frac{1}{\mathfrak{c} M}\left[\partial_{\chi} \ln \left(\frac{N}{\mathfrak{c}}\right)-M^{\mathbf{j}} \partial_{\mathbf{j}} \ln \left(\frac{N}{\mathfrak{c}}\right)\right]\right\}$ & & \\
$l^{a}$ & $\frac{1}{M N}\left[-\partial_{t}(\mathfrak{c} M)+N^{\mathbf{j}} \partial_{\mathbf{j}}(\mathfrak{c} M)\right]$ & $\partial_{\mathbf{j}} \ln (\mathfrak{c} M)$ \\
$G_{\mathbf{i}}^{a}$ & $\frac{1}{M N}\left[-\partial_{t} M^{\mathbf{i}}+\partial_{\chi} N^{\mathbf{i}}-M^{\mathbf{j}} \partial_{\mathbf{j}} N^{\mathbf{i}}+N^{\mathbf{j}} \partial_{\mathbf{j}} M^{\mathbf{i}}\right]$ & $\frac{\mathfrak{c}}{N}\left(\partial_{\mathbf{j}} N^{\mathbf{i}}\right)$ & $\frac{\mathfrak{s}}{N} \partial_{\mathbf{j}} N^{\mathbf{i}}+\frac{1}{\mathfrak{c} M} \partial_{\mathbf{j}} M^{\mathbf{i}}$
\end{tabular}

TABLE II: The algebra of the basis vectors $g_{\mathbf{A}}$. The components of the brackets enlisted in the first line along the vectors in the first column are given.

For the hypersurface-orthogonal vectors $n^{a}$ and $l^{a}$ normal fundamental scalars

$$
\begin{aligned}
\mathcal{K} & \equiv m^{d} m^{c} \tilde{\nabla}_{c} n_{d}, \\
\mathcal{L} & \equiv k^{d} k^{c} \tilde{\nabla}_{c} l_{d}
\end{aligned}
$$

can be defined. The corresponding quantities for the basis vectors $k^{a}$ and $m^{a}$ are

$$
\begin{aligned}
\mathcal{K}^{*} & \equiv l^{d} l^{c} \tilde{\nabla}_{c} k_{d}, \\
\mathcal{L}^{*} & \equiv n^{c} n^{d} \tilde{\nabla}_{c} m_{d} .
\end{aligned}
$$

Finally the two timelike vector congruences have the curvatures (nongravitational 3-dimensional accelerations):

$$
\begin{gathered}
\hat{\alpha}_{a} \equiv n^{b} \tilde{\nabla}_{b} n_{a}=\mathfrak{a}_{a}-m_{a} \mathcal{L}^{*}, \\
\hat{\alpha}_{a}^{*} \equiv k^{b} \tilde{\nabla}_{b} k_{a}=\mathfrak{a}_{a}^{*}-l_{a} \mathcal{L},
\end{gathered}
$$

the second set of expressions representing their $2+1$ decomposed form with the 2-dimensional acceleration components:

$$
\begin{aligned}
& \mathfrak{a}_{a} \equiv g_{a}^{c} n^{b} \tilde{\nabla}_{b} n_{c}, \\
& \mathfrak{a}_{a}^{*} \equiv g_{a}^{c} k^{b} \tilde{\nabla}_{b} k_{c} .
\end{aligned}
$$

Similarly, the spacelike congruences $l^{a}$ and $m^{a}$ have the 3-dimensional curvatures:

$$
\begin{gathered}
\check{\beta}_{a} \equiv l^{b} \tilde{\nabla}_{b} l_{a}=\mathfrak{b}_{a}+k_{a} \mathcal{K}^{*}, \\
\check{\beta}_{a}^{*} \equiv m^{b} \tilde{\nabla}_{b} m_{a}=\mathfrak{b}_{a}^{*}+n_{a} \mathcal{K},
\end{gathered}
$$

with the 2-dimensional "acceleration" components:

$$
\begin{aligned}
& \mathfrak{b}_{a} \equiv g_{a}^{d} c^{c} \tilde{\nabla}_{c} l_{d}, \\
& \mathfrak{b}_{a}^{*} \equiv g_{a}^{d} m^{c} \tilde{\nabla}_{c} m_{d} .
\end{aligned}
$$

With the above-introduced quantities the $2+1+1$ decomposition of the covariant derivatives of the normals to $\Sigma_{t \chi}$ in the bases they belong is

$$
\begin{aligned}
\tilde{\nabla}_{a} n_{b}= & K_{a b}+2 m_{(a} \mathcal{K}_{b)}+m_{a} m_{b} \mathcal{K}+n_{a} m_{b} \mathcal{L}^{*} \\
& -n_{a} \mathfrak{a}_{b} \\
\tilde{\nabla}_{a} l_{b}= & L_{a b}+2 k_{(a} \mathcal{L}_{b)}+k_{a} k_{b} \mathcal{L}+l_{a} k_{b} \mathcal{K}^{*} \\
& +l_{a} \mathfrak{b}_{b} \\
\tilde{\nabla}_{a} k_{b}= & K_{a b}^{*}+l_{a} \mathcal{K}_{b}^{*}+l_{b} \mathcal{L}_{a}+l_{a} l_{b} \mathcal{K}^{*}+k_{a} l_{b} \mathcal{L} \\
& -k_{a} \mathfrak{a}_{b}^{*} \\
\tilde{\nabla}_{a} m_{b}= & L_{a b}^{*}+n_{a} \mathcal{L}_{b}^{*}+n_{b} \mathcal{K}_{a}+n_{a} n_{b} \mathcal{L}^{*}+m_{a} n_{b} \mathcal{K} \\
& +m_{a} \mathfrak{b}_{b}^{*} .
\end{aligned}
$$

For deriving Eqs. (26), (27) we have also employed the second equalities (14). The structure of Eqs. (28), (29) is slightly different due to the vorticities of the vectors $k^{a}$ and $l^{a}$.

The geometric quantities defined in this subsection are not all independent. This should be obvious as the two bases are related by a Lorentz-rotation. By tedious but straightforward algebra we expressed all starry quantities in terms of unstarred ones and $\phi$ (or $\mathcal{N})$. For example the extrinsic curvatures defined with the basis vectors of the two bases are related by a rotation matrix with angle $\psi=\arccos (1 / \cosh \phi)$ as:

$$
\left(\begin{array}{c}
K_{a b}^{*} \\
L_{a b}^{*}
\end{array}\right)=\left(\begin{array}{cc}
1 / \mathfrak{c} & \mathfrak{s} / \mathfrak{c} \\
-\mathfrak{s} / \mathfrak{c} & 1 / \mathfrak{c}
\end{array}\right)\left(\begin{array}{c}
K_{a b} \\
L_{a b}
\end{array}\right)
$$

The geometric quantities characterizing the embedding are summarized on Fig. 3 while the full set of interdependencies are given them in Table

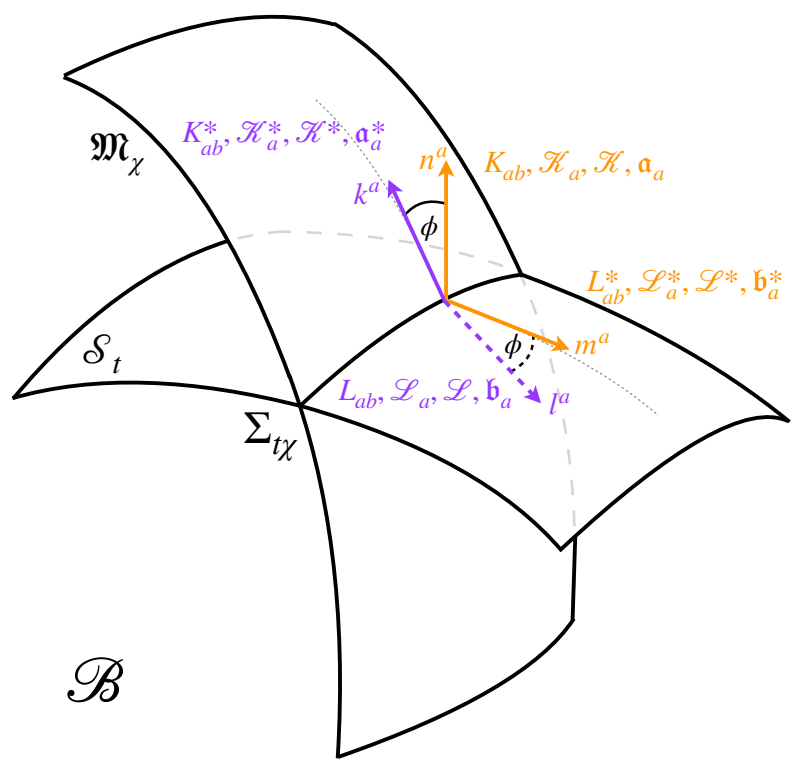

FIG. 3: The geometric embedding variables.

Note that the notations were introduced such that in the particular case $\mathcal{N}=0$ all starry quantities transform into the corresponding unstarred ones (e.g., $K_{a b}^{*}$ becomes $\left.K_{a b}\right)$. Further, as in that case the vorticities of the basis vectors $k^{a}$ and $m^{a}$ vanish, $\mathcal{L}_{a}=\mathcal{K}_{a}$ (as explored in Refs. [30, 31]) follows. 


\begin{tabular}{|l|l|}
\hline$K_{a b}^{*}=\frac{1}{\mathfrak{c}}\left(K_{a b}+\mathfrak{s} L_{a b}\right)$ & $L_{a b}^{*}=\frac{1}{c}\left(L_{a b}-\mathfrak{s} K_{a b}\right)$ \\
\hline $\mathcal{K}_{a}^{*}=\mathcal{K}_{a}+\frac{\mathfrak{s}}{c}\left(\mathfrak{a}_{a}+\mathfrak{b}_{a}\right)$ & $\mathcal{L}_{a}^{*}=\mathcal{L}_{a}+\frac{\mathfrak{s}}{c}\left(\mathfrak{a}_{a}+\mathfrak{b}_{a}\right)$ \\
\hline $\mathcal{K}^{*}=\frac{1}{c}(\mathcal{K}-\mathfrak{s} \mathcal{L})+\frac{1}{\mathfrak{c}^{2}}\left(l^{a}-\mathfrak{s} n^{a}\right) \tilde{\nabla}_{a} \phi$ & $\mathcal{L}^{*}=\frac{1}{c}(\mathfrak{s} \mathcal{K}+\mathcal{L})+\frac{1}{\mathfrak{c}^{2}}\left(\mathfrak{s} l^{a}+n^{a}\right) \nabla_{a} \phi$ \\
\hline $\mathfrak{a}_{a}^{*}=\mathfrak{a}_{a}+\frac{\mathfrak{s}}{\mathfrak{c}}\left(\mathcal{K}_{a}-\mathcal{L}_{a}\right)=\mathfrak{a}_{a}-\frac{\mathfrak{s}}{\mathfrak{c}} D_{a} \phi$ & $\mathfrak{b}_{a}^{*}=\mathfrak{b}_{a}+\frac{\mathfrak{s}}{\mathfrak{c}}\left(\mathcal{L}_{a}-\mathcal{K}_{a}\right)=\mathfrak{b}_{a}+\frac{\mathfrak{s}}{\mathfrak{c}} D_{a} \phi$ \\
\hline
\end{tabular}

TABLE III: The relations among starred and unstarred geometric quantities characterizing the embedding of $\Sigma_{t \chi}$.

\section{KINEMATICS AND GEOMETRIC EMBEDDING}

In this section we establish the relations of the temporal and spatial derivatives of the metric variables $\left\{g_{a b}, M^{a}, M\right\}$ to the geometric quantities $\left\{K^{a b}, \mathcal{K}_{a}, \mathcal{K}\right\},\left\{L^{a b}, \mathcal{L}_{a}, \mathcal{L}\right\},\left\{K^{* a b}, \mathcal{K}_{a}^{*}, \mathcal{K}^{*}\right\}$ and $\left\{L^{* a b}, \mathcal{L}_{a}^{*}, \mathcal{L}^{*}\right\}$ characterizing the embedding. These will be used later in the derivation of the Hamiltonian formulation of GR from the Einstein-Hilbert action.

Bearing in mind that both the coordinate derivatives along time and $\chi$ and the extrinsic curvatures are projected Lie derivatives, we find for the extrinsic curvature in the two bases

$$
\begin{aligned}
K_{a b} & =\frac{1}{N}\left[\frac{1}{2} \partial_{t} g_{a b}-D_{(a} N_{b)}\right]-\frac{\mathfrak{s}}{M \mathfrak{c}}\left[\frac{1}{2} \partial_{\chi} g_{a b}-D_{(a} M_{b)}\right] \\
L_{a b}^{*} & =\frac{1}{M}\left[\frac{1}{2} \partial_{\chi} g_{a b}-D_{(a} M_{b)}\right]
\end{aligned}
$$

and

$$
\begin{aligned}
L_{a b} & =\frac{\mathfrak{s}}{N}\left[\frac{1}{2} \partial_{t} g_{a b}-D_{(a} N_{b)}\right]+\frac{1}{M \mathfrak{c}}\left[\frac{1}{2} \partial_{\chi} g_{a b}-D_{(a} M_{b)}\right] \\
K_{a b}^{*} & =\frac{\mathfrak{c}}{N}\left[\frac{1}{2} \partial_{t} g_{a b}-D_{(a} N_{b)}\right]
\end{aligned}
$$

respectively. Only $L_{a b}^{*}$ is free from time derivatives of the induced metric, hence nondynamical.

In order to establish the relation of the rest of the geometric variables with time and $\chi$-derivatives of the metric variables we employ the following identity holding for all vectors $V_{\mathbf{I}}$ for which $\tilde{g}\left(V_{\mathbf{I}}, V_{\mathbf{J}}\right)=$ constant:

$$
\begin{aligned}
\tilde{g}\left(V_{\mathbf{A}}, \tilde{\nabla}_{V_{\mathbf{B}}} V_{\mathbf{C}}\right)= & \tilde{g}\left(\left[V_{\mathbf{A}}, V_{\mathbf{B}}\right], V_{\mathbf{C}}\right) \\
& -\tilde{g}\left(V_{\mathbf{C}}, \tilde{\nabla}_{V_{\mathbf{A}}} V_{\mathbf{B}}\right) .
\end{aligned}
$$

First we apply this identity for the case $V_{\mathbf{B}}=V_{\mathbf{C}}$, such that the last term vanishes. Then for the basis vectors $f_{\mathbf{A}}$ and $g_{\mathbf{A}}$ perpendicular to $\Sigma_{t \chi}$ the lefthand sides are the accelerations $\hat{\alpha}_{a}=\tilde{g}\left(f_{\mathbf{A}}, \tilde{\nabla}_{\mathbf{n}} \mathbf{n}\right) f_{a}^{\mathbf{A}}$, $\hat{\alpha}_{a}^{*}=\tilde{g}\left(g_{\mathbf{A}}, \tilde{\nabla}_{\mathbf{k}} \mathbf{k}\right) g_{a}^{\mathbf{A}}, \check{\beta}_{a}=\tilde{g}\left(g_{\mathbf{A}}, \tilde{\nabla}_{\mathbf{l}} \mathbf{l}\right) g_{a}^{\mathbf{A}}$ and $\check{\beta}_{a}^{*}=$ $\tilde{g}\left(f_{\mathbf{A}}, \tilde{\nabla}_{\mathbf{m}} \mathbf{m}\right) f_{a}^{\mathbf{A}}$. Calculating the right-hand sides by exploring the specific components of the Lie brackets given in Tables $\Pi$ and $\Pi$ and comparing the resulting expressions with the decompositions given in Eqs. (20),
(21), (23) and (24) we obtain the 2-dimensional accelerations as projected covariant derivatives

$$
\begin{aligned}
\mathfrak{a}_{a} & =D_{a}(\ln N), \\
\mathfrak{b}_{a}^{*} & =-D_{a}(\ln M), \\
\mathfrak{b}_{a} & =-D_{a} \ln (\mathfrak{c} M), \\
\mathfrak{a}_{a}^{*} & =D_{a}\left(\ln \frac{N}{\mathfrak{c}}\right),
\end{aligned}
$$

while the normal fundamental scalars emerge as

$$
\begin{aligned}
\mathcal{K} & =\frac{1}{M N}\left[\partial_{t} M-\partial_{\chi} \mathcal{N}-N^{a} D_{a} M+M^{a} D_{a} \mathcal{N}\right] \\
\mathcal{L}^{*} & =-\frac{1}{M}\left[\partial_{\chi}(\ln N)-M^{a} D_{a}(\ln N)\right], \\
\mathcal{L} & =-\mathcal{S}-\frac{1}{\mathfrak{c} M}\left[\partial_{\chi} \ln \left(\frac{N}{\mathfrak{c}}\right)-M^{a} D_{a} \ln \left(\frac{N}{\mathfrak{c}}\right)\right], \\
\mathcal{K}^{*} & =\frac{1}{M N}\left[\partial_{t}(\mathfrak{c} M)-N^{a} D_{a}(\mathfrak{c} M)\right],
\end{aligned}
$$

with

$$
\begin{aligned}
\mathcal{S}= & \partial_{t}\left(\frac{\mathfrak{s}}{N}\right)-N^{a} D_{a}\left(\frac{\mathfrak{s}}{N}\right) \\
& +\frac{\mathfrak{s}}{N}\left[\partial_{t} \ln (M N)-N^{a} D_{a} \ln (M N)\right]
\end{aligned}
$$

(an expression which vanishes for orthogonal foliations).

Next we apply the identity (33) for $n^{b} \tilde{\nabla}_{b} m_{a}=$ $\tilde{g}\left(f_{\mathbf{A}}, \tilde{\nabla}_{\mathbf{n}} \mathbf{m}\right) f_{a}^{\mathbf{A}}$ and $l^{b} \tilde{\nabla}_{b} k_{a}=\tilde{g}\left(g_{\mathbf{A}}, \tilde{\nabla}_{\mathbf{l}} \mathbf{k}\right) g_{a}^{\mathbf{A}}$, respectively, obtaining for the $\Sigma_{t \chi}$ projections

$$
\begin{aligned}
\mathcal{L}_{a}^{*} & =\mathcal{K}_{a}+\frac{M}{N} D_{a}\left(\frac{\mathcal{N}}{M}\right), \\
\mathcal{K}_{a}^{*} & =\mathcal{L}_{a}-\frac{N}{\mathfrak{c}^{2} M} D_{a}\left(\frac{\mathfrak{s c} M}{N}\right) .
\end{aligned}
$$

These can be also derived from the expressions given in Table III together with Eqs. (15) and (34). Now we have everything at hand to derive the relation of the normal fundamental forms and metric derivatives. For this we rewrite

$$
\begin{aligned}
\mathcal{K}_{a} & =-g_{a b}[m, n]^{b}-\mathcal{L}_{a}^{*}, \\
\mathcal{L}_{a} & =-g_{a b}[k, l]^{b}-\mathcal{K}_{a}^{*},
\end{aligned}
$$

employ the algebras of the basis vectors $f_{\mathbf{A}}$ and $g_{\mathbf{A}}$ given in Tables I and II] respectively, together with Eqs. (37) 
and (38), to obtain

$$
\begin{aligned}
\mathcal{K}^{a}= & \frac{1}{2 M N}\left(\partial_{t} M^{a}-\partial_{\chi} N^{a}-N^{b} D_{b} M^{a}+M^{b} D_{b} N^{a}\right) \\
& -\frac{M}{2 N} D^{a}\left(\frac{\mathcal{N}}{M}\right), \\
\mathcal{L}^{a}= & \frac{1}{2 M N}\left(\partial_{t} M^{a}-\partial_{\chi} N^{a}-N^{b} D_{b} M^{a}+M^{b} D_{b} N^{a}\right) \\
& +\frac{N}{2 \mathfrak{c}^{2} M} D^{a}\left(\frac{\mathfrak{s c} M}{N}\right), \\
\mathcal{K}^{* a}= & \frac{1}{2 M N}\left(\partial_{t} M^{a}-\partial_{\chi} N^{a}-N^{b} D_{b} M^{a}+M^{b} D_{b} N^{a}\right) \\
& -\frac{N}{2 \mathfrak{c}^{2} M} D^{a}\left(\frac{\mathfrak{s c} M}{N}\right), \\
\mathcal{L}^{* a}= & \frac{1}{2 M N}\left(\partial_{t} M^{a}-\partial_{\chi} N^{a}-N^{b} D_{b} M^{a}+M^{b} D_{b} N^{a}\right) \\
& +\frac{M}{2 N} D^{a}\left(\frac{\mathcal{N}}{M}\right) .
\end{aligned}
$$

Note that the metric derivatives are related to the normal fundamental vectors, rather then forms.

From the results of this and of the previous section we can conclude that the independent metric variables with dynamical role are $\left\{g_{a b}, M^{a}, M\right\}$ while the embedding variables $\left\{K^{a b}, \mathcal{K}_{a}, \mathcal{K}\right\}$ carry information about their temporal evolution. The extrinsic curvature $L_{a b}^{*}$ being the only one, which contains no time derivatives, it plays a nondynamical role. Hence we chose the variables emerging in the $f_{\mathbf{A}}$ basis as independent, $\left\{K^{a b}, \mathcal{K}_{a}, \mathcal{K}\right\}$ representing momenta, while $\left\{L^{* a b}, \mathcal{L}^{*}\right\}$ merely spatial derivatives. All other embedding variables can be expressed in terms of this set.

\section{HAMILTONIAN FORMALISM IN GENERAL RELATIVITY}

In this section we present the $2+1+1$ decomposed Hamiltonian formalism in general relativity. As discussed earlier, we employ the $f_{\mathbf{A}}$ basis in the decomposition.

\section{A. The $2+1+1$ decomposition of the Einstein-Hilbert action}

We define the 2-dimensional Riemann tensor $R_{a b c d}$ of the metric induced in $\Sigma_{t \chi}$ as

$$
R_{a b c d} V^{b}=\left(D_{c} D_{d}-D_{d} D_{c}\right) V_{a},
$$

which written in terms of the geometric quantities arising in the $2+1+1$ decomposition and of the 4-dimensional Riemann tensor leads to the following Gauss-type identity:

$$
R_{a b c d}=g_{a}^{i} g_{b}^{j} g_{c}^{k} g_{d}^{l} \tilde{R}_{i j k l}+2\left(L_{a[c}^{*} L_{d] b}^{*}-K_{a[c} K_{d] b}\right)
$$

The extrinsic curvatures are those appearing in the $f_{\mathbf{A}}$ basis. Twice contracting this leads to

$$
R=g^{i k} g^{j l} \tilde{R}_{i j k l}+\left(L^{*}\right)^{2}-K^{2}-L_{a b}^{*} L^{* a b}+K_{a b} K^{a b} .
$$

The first term on the right-hand side is decomposed as

$$
\begin{aligned}
g^{i k} g^{j l} \tilde{R}_{i j k l}= & \tilde{R}+2\left(n^{j} n^{l}-m^{j} m^{l}\right) \tilde{R}_{j l} \\
& -2 n^{i} m^{j} n^{k} m^{l} \tilde{R}_{i j k l}
\end{aligned}
$$

where

$$
\begin{aligned}
m^{i} n^{j} n^{k} m^{l} \tilde{R}_{i j k l}= & \mathcal{K}^{k}\left(2 \mathcal{L}_{k}^{*}+\mathcal{K}_{k}\right)-\left(\mathcal{L}^{*}\right)^{2}+(\mathcal{K})^{2} \\
& +\tilde{\mathfrak{L}}_{\mathbf{m}} \mathcal{L}^{*}+\tilde{\mathfrak{L}}_{\mathbf{n}} \mathcal{K}-\frac{D^{i} N D_{i} M}{N M} \\
n^{j} n^{l} \tilde{R}_{j l}= & -K^{l b} K_{b l}-\mathcal{L}^{*} L^{*}-2 \mathcal{K}^{b} \mathcal{K}_{b}-(\mathcal{K})^{2} \\
& +\left(\mathcal{L}^{*}\right)^{2}-\tilde{\mathfrak{L}}_{\mathbf{n}} K-\tilde{\mathfrak{L}}_{\mathbf{n}} \mathcal{K}-\tilde{\mathfrak{L}}_{\mathbf{m}} \mathcal{L}^{*} \\
& +\frac{D_{b} D^{b} N}{N}+\frac{D^{b} N D_{b} M}{N M}, \\
m^{j} m^{l} \tilde{R}_{j l}= & -L^{* l b} L_{b l}^{*}+2 \mathcal{K}_{l} \mathcal{L}^{* l}-\left(\mathcal{L}^{*}\right)^{2}+(\mathcal{K})^{2} \\
& +\mathcal{K} K^{\tilde{\mathfrak{L}}} \mathcal{K}_{\mathbf{K}}-\tilde{\mathfrak{L}}_{\mathbf{m}} L^{*}+\tilde{\mathfrak{L}} \mathbf{m} \mathcal{L}^{*} \\
& -\left(\frac{D_{b} D^{b} M}{M}+\frac{D^{b} M D_{b} N}{N M}\right) .
\end{aligned}
$$

In order to prove the above expressions we have explored the useful identities

$$
\begin{gathered}
\tilde{\nabla}_{a} \mathfrak{a}^{a}=\frac{D_{a} D^{a} N}{N}+\frac{D^{a} N D_{a} M}{N M}, \\
\tilde{\nabla}_{a} \mathfrak{b}^{* a}=-\left(\frac{D_{a} D^{a} M}{M}+\frac{D^{a} M D_{a} N}{N M}\right),
\end{gathered}
$$

and

$$
\tilde{\nabla}_{a} n^{a}=K+\mathcal{K}, \quad \tilde{\nabla}_{a} m^{a}=L^{*}-\mathcal{L}^{*} .
$$

With these the twice contracted Gauss relation becomes ${ }^{2}$

$$
\begin{aligned}
R= & \tilde{R}-K^{2}-K_{a b} K^{a b}+\left(L^{*}\right)^{2}+L_{a b}^{*} L^{* a b}-2 \mathcal{K}^{b} \mathcal{K}_{b} \\
& -2 \mathcal{K}(K+\mathcal{K})+2 \mathcal{L}^{*}\left(\mathcal{L}^{*}-L^{*}\right) \\
& -2 \tilde{\mathfrak{L}}_{\mathbf{n}}(K+\mathcal{K})+2 \tilde{\mathfrak{L}}_{\mathbf{m}}\left(L^{*}-\mathcal{L}^{*}\right) \\
& +2\left[\frac{D_{a} D^{a} N}{N}+\frac{D_{a} D^{a} M}{M}+\frac{D^{a} M D_{a} N}{N M}\right] .
\end{aligned}
$$

Noting that $\sqrt{-\tilde{g}}=N M \sqrt{g}$ the Einstein-Hilbert action

$$
\begin{aligned}
S_{E H} & =\int d t \int d \chi \int_{\Sigma_{t \chi}} d^{2} x \mathcal{L}^{G}, \\
\mathcal{L}^{G} & =\sqrt{-\tilde{g} \tilde{R}}
\end{aligned}
$$

\footnotetext{
2 By suitably transforming the Lie derivatives this expression becomes identical with the one obtained for orthogonal double foliations, Eq. (A1) of Ref. [31], after correcting the coefficient of $\left(L_{a b}^{*} L^{* a b}-K_{a b} K^{a b}\right)$ from -3 to +1 in the latter.
} 
can be $2+1+1$ decomposed as follows:

$$
\begin{aligned}
& \mathcal{L}^{G}\left[\left\{g_{a b}, M^{a}, M\right\} ;\left\{K^{a b}, \mathcal{K}_{a}, \mathcal{K}\right\} ;\left\{L^{* a b}, \mathcal{L}^{*}\right\} ;\left\{N, N^{a}, \mathcal{N}\right\}\right] \\
= & N M \sqrt{g}\left\{R+K_{a b} K^{a b}+K^{2}-\left(L^{*}\right)^{2}-L_{a b}^{*} L^{* a b}\right. \\
& +2 \mathcal{K}^{a} \mathcal{K}_{a}+2 \mathcal{K}(K+\mathcal{K})-2 \mathcal{L}^{*}\left(\mathcal{L}^{*}-L^{*}\right) \\
& +2 \tilde{\mathfrak{L}}_{\mathbf{n}}(K+\mathcal{K})-2 \tilde{\mathfrak{L}}_{\mathbf{m}}\left(L^{*}-\mathcal{L}^{*}\right)-2\left[N^{-1} D_{a} D^{a} N\right. \\
& \left.\left.+M^{-1} D_{a} D^{a} M+(N M)^{-1} D^{a} M D_{a} N\right]\right\} .
\end{aligned}
$$

This form of the action is ready to be employed in the Legendre transformation.

\section{B. The Legendre transformation}

The action (50) has to be further transformed in order to derive the canonical momenta. By employing

$$
\begin{aligned}
\tilde{\mathfrak{L}}_{\mathbf{n}}(K+\mathcal{K}) & =\tilde{\nabla}_{a}\left[n^{a}(K+\mathcal{K})\right]-(K+\mathcal{K})^{2}, \\
\tilde{\mathfrak{L}}_{\mathbf{m}}\left(L^{*}-\mathcal{L}^{*}\right) & =\tilde{\nabla}_{a}\left[m^{a}\left(L^{*}-\mathcal{L}^{*}\right)\right]-\left(L^{*}-\mathcal{L}^{*}\right)^{2},
\end{aligned}
$$

we rewrite it in a form explicitly containing all boundary terms (total divergences):

$$
\begin{aligned}
\mathcal{L}^{G}= & N M \sqrt{g}\left\{R+K_{a b} K^{a b}-K^{2}-2 K \mathcal{K}+2 \mathcal{K}^{a} \mathcal{K}_{a}\right. \\
& -L_{a b}^{*} L^{* a b}+L^{* 2}-2 \mathcal{L}^{*} L^{*}+2(N M)^{-1} D^{a} M D_{a} N \\
& \left.-2 \tilde{\nabla}_{a}\left[\hat{\alpha}^{a}-\check{\beta}^{* a}-n^{a} K+m^{a} L^{*}\right]\right\} .
\end{aligned}
$$

This contains expressions of the metric variables $\left\{g_{a b}, M^{a}, M\right\}$, geometric quantities $\left\{K^{a b}, \mathcal{K}_{a}, \mathcal{K}\right\}$ containing their time derivatives, purely spatial derivatives $\left\{L^{* a b}, \mathcal{L}^{*}\right\}$ [see Eqs. (31), (32)]; the lapse and shift components $\left\{N, N^{a}, \mathcal{N}\right\}$ and total divergences. The latter do not contribute to the dynamics, hence can be omitted when calculating the canonical momenta:

$$
\begin{aligned}
\pi^{a b} & =\frac{\partial \mathcal{L}^{G}}{\partial \dot{g}^{a b}}=\sqrt{g} M\left[K^{a b}-g^{a b}(K+\mathcal{K})\right], \\
p_{a} & =\frac{\partial \mathcal{L}^{G}}{\partial \dot{M}^{a}}=2 \sqrt{g} \mathcal{K}_{a} \\
p & =\frac{\partial \mathcal{L}^{G}}{\partial \dot{M}}=-2 \sqrt{g} K
\end{aligned}
$$

With them we rewrite the Lagrangian density once again with the aim to manifestly obtain the Liouvilleform. This is achieved by transforming (the double of) the terms quadratic in the set $\left\{K^{a b}, \mathcal{K}_{a}, \mathcal{K}\right\}$ in the Lagrangian density into expressions linear in the time derivatives of $\left\{g_{a b}, M^{a}, M\right\}$. After extensive calculations we obtain

$$
\begin{aligned}
\mathcal{L}^{G}= & \pi^{a b} \dot{g}_{a b}+p_{a} \dot{M}^{a}+p \dot{M}-\mathcal{H}^{G} \\
& +\mathcal{L}_{t}^{G}+\mathcal{L}_{\chi}^{G}+\mathcal{L}_{D}^{G},
\end{aligned}
$$

where

$$
\mathcal{H}^{G}=N \mathcal{H}_{\perp}^{G}+N^{a} \mathcal{H}_{a}^{G}+\mathcal{N} \mathcal{H}_{\mathcal{N}}^{G}
$$

is the vacuum gravitational Hamiltonian density in GR, a linear combination of the products of the Lagrange multipliers $\left\{N, N^{a}, \mathcal{N}\right\}$ with the Hamiltonian constraint ${ }^{3}$ :

$$
\begin{aligned}
\mathcal{H}_{\perp}^{G}= & \sqrt{g}\left\{M \left(-R-3 L^{* a b} L_{a b}^{*}+L^{* 2}+K_{a b} K^{a b}\right.\right. \\
& \left.+2 \mathcal{K}_{a} \mathcal{K}^{a}-K^{2}-2 K \mathcal{K}\right)+2 g^{a b} \partial_{\chi} L_{a b}^{*} \\
& \left.-2 M^{a} D_{a} L^{*}-4 L_{a b}^{*} D^{a} M^{b}+2 D^{a} D_{a} M\right\},
\end{aligned}
$$

("angular") diffeomorphism constraints along $\Sigma_{t \chi}$ :

$$
\begin{aligned}
\mathcal{H}_{a}^{G}= & -2 \sqrt{g}\left\{D_{b}\left[K^{b}{ }_{a} M-M g^{b}{ }_{a}(K+\mathcal{K})\right]+K D_{a} M\right. \\
& \left.+\mathcal{K}_{a} M L^{*}+\partial_{\chi} \mathcal{K}_{a}-M^{b} D_{b} \mathcal{K}_{a}-\mathcal{K}_{b} D_{a} M^{b}\right\},(56)
\end{aligned}
$$

and along $m^{a}$ ("radial" diffeomorphism constraint):

$$
\begin{aligned}
\mathcal{H}_{\mathcal{N}}^{G}= & -2 \sqrt{g}\left[M\left(L^{*} \mathcal{K}-L_{a b}^{*} K^{a b}\right)+M D_{a} \mathcal{K}^{a}\right. \\
& \left.+2 \mathcal{K}^{a} D_{a} M-\partial_{\chi} K+M^{a} D_{a} K\right],
\end{aligned}
$$

respectively, finally the terms

$$
\begin{aligned}
\mathcal{L}_{t}^{G}= & 2 \partial_{t}[\sqrt{g} M(K+\mathcal{K})] \\
\mathcal{L}_{\chi}^{G}= & 2 \partial_{\chi}\left[\sqrt{g}\left(N \mathcal{L}^{*}-N_{a} \mathcal{K}^{a}-\mathcal{N K}\right)\right], \\
\mathcal{L}_{D}^{G}= & -2 \sqrt{g} D_{a}\left[M D^{a} N+N^{b}\left(M K^{a}{ }_{b}-M^{a} \mathcal{K}_{b}\right)\right. \\
& \left.+N M^{a} \mathcal{L}^{*}+\mathcal{N}\left(M \mathcal{K}^{a}-M^{a} \mathcal{K}\right)\right]
\end{aligned}
$$

are boundary contributions. Employing the inverses

$$
\begin{aligned}
K^{a b} & =\frac{1}{M \sqrt{g}}\left(\pi^{a b}-\frac{\pi}{2} g^{a b}\right)-\frac{p}{4 \sqrt{g}} g^{a b}, \\
\mathcal{K}_{a} & =\frac{1}{2 \sqrt{g}} p_{a}, \\
\mathcal{K} & =\frac{1}{4 \sqrt{g}}\left(p-\frac{2 \pi}{M}\right),
\end{aligned}
$$

of Eqs. (52) and introducing Lie derivatives by remembering that the momenta are tensor densities ${ }^{4}$, all expressions can be rewritten in terms of the set of canonical coordinates $\left\{g_{a b}, M^{a}, M\right\}$ and canonical momenta $\left\{\pi^{a b}, p_{a}, p\right\}$ as follows:

$$
\begin{aligned}
\mathcal{H}_{\perp}^{G}= & \sqrt{g}\left[-M\left(R+3 L^{* a b} L_{a b}^{*}-L^{* 2}\right)+2 D^{a} D_{a} M\right. \\
& \left.+2 g^{a b}\left(\partial_{\chi}-\mathfrak{L}_{\mathbf{M}}\right) L_{a b}^{*}\right]+\frac{1}{M \sqrt{g}}\left(\pi_{a b} \pi^{a b}-\frac{\pi^{2}}{2}\right) \\
& +\frac{M}{\sqrt{g}}\left(\frac{1}{2} p_{a} p^{a}+\frac{1}{8} p^{2}-\frac{\pi p}{2 M}\right) \\
\mathcal{H}_{a}^{G}= & -2 D_{b} \pi_{a}^{b}+p D_{a} M-\left(\partial_{\chi}-\mathfrak{L}_{\mathbf{M}}\right) p_{a} \\
\mathcal{H}_{\mathcal{N}}^{G}= & 2 L_{a b}^{*} \pi^{a b}-2 p^{a} D_{a} M-M D_{a} p^{a} \\
& -\left(\partial_{\chi}-\mathfrak{L}_{\mathbf{M}}\right) p .
\end{aligned}
$$

\footnotetext{
3 This expression reproduces Eq. (13a) of Ref. [31] after correcting the misprints in the signs of the second and third term.

${ }^{4}$ For an arbitrary tensor density $\mathcal{F}=f \sqrt{g}$ (where $f$ is a tensor) its Lie derivative along $M^{a}$ is $\mathfrak{L}_{\mathbf{M}} \mathcal{F}=D_{a}\left(\mathcal{F} M^{a}\right)$.
} 
The constraints (60) and (61) fully agree with the respective ones of Ref. [31], while the last constraint (62) is new, emerging only in the nonorthogonal double foliation.

Similarly, the boundary terms emerge as:

$$
\begin{aligned}
\mathcal{L}_{t}^{G}= & -\partial_{t}\left(\pi+\frac{M p}{2}\right) \\
\mathcal{L}_{\chi}^{G}= & \partial_{\chi}\left[2 \sqrt{g} N \mathcal{L}^{*}-N_{a} p^{a}+\mathcal{N}\left(\frac{\pi}{M}-\frac{p}{2}\right)\right], \\
\mathcal{L}_{D}^{G}= & -D_{a}\left\{2 \sqrt{g}\left(M D^{a} N+N M^{a} \mathcal{L}^{*}\right)\right. \\
& +N^{b}\left[2 \pi^{a}{ }_{b}-\left(\pi+\frac{M p}{2}\right) g_{b}^{a}-M^{a} p_{b}\right] \\
& \left.+\mathcal{N}\left[M p^{a}+M^{a}\left(\frac{\pi}{M}-\frac{p}{2}\right)\right]\right\} .
\end{aligned}
$$

Unlike the constraints, the boundary terms on the spatial infinity are modified by new terms proportional to $\mathcal{N}$.

Following the same steps, the time derivatives of the canonical coordinates can be expressed from Eqs. (31), (35) and (39) as follows:

$$
\begin{aligned}
\dot{g}_{a b}= & \frac{N}{M \sqrt{g}}\left[2 \pi_{a b}-\left(\pi+\frac{M p}{2}\right) g_{a b}\right] \\
& +\mathfrak{L}_{\mathbf{N}} g_{a b}+\frac{\mathcal{N}}{M}\left(\partial_{\chi}-\mathfrak{L}_{\mathbf{M}}\right) g_{a b}, \\
\dot{M}^{a}= & \frac{M N}{\sqrt{g}} p^{a}+\left(\partial_{\chi}-\mathfrak{L}_{\mathbf{M}}\right) N^{a}+M D^{a} \mathcal{N}-\mathcal{N} D^{a} M, \\
\dot{M}= & \frac{M N}{4 \sqrt{g}}\left(p-\frac{2 \pi}{M}\right)+\mathfrak{L}_{\mathbf{N}} M+\left(\partial_{\chi}-\mathfrak{L}_{\mathbf{M}}\right) \mathcal{N} .
\end{aligned}
$$

These are but the evolution equations of the canonical coordinates, thus half of the canonical equations. Note that all of them contain terms with $\mathcal{N}$, the rest of the terms agreeing with those derived for the orthogonal case in Ref. [31.

\section{Canonical equations}

In order to simplify the presentation, we introduce the notations $g^{A} \equiv\left\{g_{a b}, M^{a}, M\right\}$ for the set of canonical coordinates, $\pi_{A} \equiv\left\{\pi^{a b}, p_{a}, p\right\}$ for the canonical momenta, and $y=\left\{y^{1}, y^{2}\right\}$ for the coordinates adapted to $\Sigma_{t \chi}$. The $2+1+1$ decomposed Hamiltonian identified in the previous subsection is

$$
H^{G}=\int d \chi \int d y \mathcal{H}^{G}(\chi, y) .
$$

Time derivatives of the canonical variables emerge as functional derivatives of the Hamiltonian:

$$
\begin{aligned}
\dot{g}^{A} & =\frac{\delta H^{G}}{\delta \pi_{A}(\chi, y)}, \\
\dot{\pi}_{A} & =-\frac{\delta H^{G}}{\delta g^{A}(\chi, y)} .
\end{aligned}
$$

It can be verified that Eq. (66) reproduces the set of equations of motion (64). Next we calculate Eq. (67) in detail. Lengthy but straightforward computations lead to the second set of canonical equations:

$$
\begin{aligned}
\dot{\pi}^{a b}= & N \mathcal{S}^{a b}+N \mathcal{V}^{a b}-N M \sqrt{g} \mathcal{L}^{*}\left(L^{* a b}-L^{*} g^{a b}\right) \\
& +\sqrt{g}\left[M D^{a} D^{b} N-g^{a b} M D^{c} D_{c} N\right. \\
& \left.-g^{a b}\left(D_{c} N\right)\left(D^{c} M\right)+g^{a b}\left(\partial_{\chi}-\mathfrak{L}_{\mathbf{M}}\right)\left(N \mathcal{L}^{*}\right)\right] \\
& +\mathfrak{L}_{\mathbf{N}} \pi^{a b}-\left[\frac{\mathcal{N} \pi^{a b}}{M^{2}}\left(\partial_{\chi}-\mathfrak{L}_{\mathbf{M}}\right)+\mathcal{N} p^{(a} D^{b)}\right] M \\
& +\left[\frac{\pi^{a b}}{M}\left(\partial_{\chi}-\mathfrak{L}_{\mathbf{M}}\right)+M p^{(a} D^{b)}\right] \mathcal{N} \\
& +\frac{\mathcal{N}}{M}\left(\partial_{\chi}-\mathfrak{L}_{\mathbf{M}}\right) \pi^{a b}, \\
\dot{p}_{a}= & N \mathcal{V}_{a}-2 \sqrt{g}\left[L_{b a}^{*} D^{b} N+D_{a}\left(N \mathcal{L}^{*}\right)\right]+\mathfrak{L}_{\mathbf{N}} p_{a} \\
& -\frac{2 \mathcal{N}}{M} D^{b} \pi_{b a}+\frac{2 \mathcal{N}}{M^{2}} \pi_{b a} D^{b} M \\
& +\left(p g_{a b}-\frac{2}{M} \pi_{a b}\right) D^{b} \mathcal{N}, \\
\dot{p}= & N \mathcal{S}+N \mathcal{V}-2 \sqrt{g}\left(L^{*} \mathcal{L}^{*}+D_{a} D^{a} N\right)+\mathfrak{L}_{\mathbf{N}} p \\
& +\mathcal{N}\left(\frac{2}{M} \pi^{a b} L_{a b}^{*}-D_{a} p^{a}\right)-2 p^{a} D_{a} \mathcal{N} .
\end{aligned}
$$

Here $\mathcal{S}^{a b}$ and $\mathcal{S}$ are

$$
\begin{aligned}
\mathcal{S}^{a b}= & -\frac{2}{M \sqrt{g}}\left(\pi^{a}{ }_{c} \pi^{b c}-\frac{\pi}{2} \pi^{a b}\right) \\
& +\frac{1}{2 M \sqrt{g}}\left(\pi_{c d} \pi^{c d}-\frac{\pi^{2}}{2}\right) g^{a b} \\
& -\frac{M}{4 \sqrt{g}} g^{a b}\left(\frac{\pi p}{M}-p_{c} p^{c}-\frac{p^{2}}{4}\right) \\
& +\frac{1}{2 \sqrt{g}}\left(p \pi^{a b}+M p^{a} p^{b}\right),
\end{aligned}
$$

$$
\begin{aligned}
\mathcal{S}= & \frac{1}{\sqrt{g} M^{2}}\left(\pi_{a b} \pi^{a b}-\frac{\pi^{2}}{2}\right) \\
& -\frac{1}{2 \sqrt{g}}\left(p_{a} p^{a}+\frac{p^{2}}{4}\right),
\end{aligned}
$$

while $\mathcal{V}^{a b}, \mathcal{V}_{a}$, and $\mathcal{V}$ represent the tensorial, vectorial, and scalar projections of the force term of the $(s+1)$ dimensional scalar curvature potential, given in Ref. [31]:

$$
\begin{aligned}
\mathcal{V}^{a b}= & -M \sqrt{g}\left(G^{a b}+2 L^{* a c} L^{* b}{ }_{c}-L^{*} L^{* a b}\right) \\
& +\frac{M}{2} \sqrt{g}\left(3 L^{* c d} L_{c d}^{*}-L^{* 2}\right) g^{a b} \\
& +\sqrt{g}\left(g^{a c} g^{b d}-g^{a b} g^{c d}\right)\left(\partial / \partial \chi-\mathfrak{L}_{\mathbf{N}}\right) L_{c d}^{*} \\
& +\sqrt{g}\left(D^{a} D^{b} M-g^{a b} D^{c} D_{c} M\right),
\end{aligned}
$$




$$
\begin{aligned}
& \mathcal{V}_{a}=-2 \sqrt{g}\left(D^{b} L_{b a}^{*}-D_{b} L^{*}\right) \\
& \mathcal{V}=\sqrt{g}\left(R+L_{a b}^{*} L^{* a b}-L^{* 2}\right) .
\end{aligned}
$$

The canonical equations given by Eqs. (64) and 68, 70. are the generalizations of Eqs. (29a-30c) of Ref. [31] for the case of nonorthogonal double foliation of the 4dimensional spacetime.

\section{GAUGE TRANSFORMATIONS AND FIXING IN PERTURBATIONS OF SPHERICALLY SYMMETRIC, STATIC BLACK HOLES IN GENERIC SCALAR-TENSOR THEORIES}

In GR the perturbations of the spherically symmetric, static spacetime have been discussed both for the odd [44] and for the even parity sectors [45]. The 10 metric functions were analyzed by employing a $2+1+1$ decomposition based on the temporal and radial direction and a further decomposition of the metric perturbation into spherical harmonics and its derivatives. As result of the choice of polar coordinates, 8 metric perturbations survived, however suitably adapting the remaining diffeomorphism freedom, the odd sector has been expressed in terms of 2 , the even sector in terms of 4 , respectively [44].

When we consider generic scalar perturbations of spherically symmetric, static black holes in those scalartensor gravitational theories, which avoid Ostrogradsky instabilities, an additional scalar variable pops in, further complicating the gauge choice. We address this problem in this section.

All background quantities will be denoted by an overbar and the respective perturbed quantities will be written as $N=\bar{N}+\delta N$, etc. Due to the high degree of symmetry of the background we could assume both the temporal and spatial evolutions perpendicular to $\Sigma_{t \chi}$ (hence $\left.\bar{N}^{a}=\bar{M}^{a}=0\right)$, the foliations perpendicular $(\overline{\mathcal{N}}=0)$ and radial unitary gauge $(\bar{\phi}=\bar{\phi}(\chi))$, thus the scalar depending only on the radial coordinate $\chi$. Hence the perturbed metric to first order becomes

$$
\begin{aligned}
d s^{2}= & -\left(\bar{N}^{2}+2 \bar{N} \delta N\right) d t^{2}+2 \bar{M} \delta \mathcal{N} d t d \chi \\
& +2 \delta N_{a} d t d x^{a}+\left(\bar{g}_{a b}+\delta g_{a b}\right) d x^{a} d x^{b} \\
& +2 \delta M_{a} d x^{a} d \chi+\left(\bar{M}^{2}+2 \bar{M} \delta M\right) d \chi^{2}
\end{aligned}
$$

while the scalar field changes as

$$
\phi=\bar{\phi}(\chi)+\delta \phi
$$

Helmholtz-like decompositions on spherically symmetric background hold for both vectors:

$$
V_{a}=\bar{D}_{a} V_{\text {rotfree }}+E^{b}{ }_{a} \bar{D}_{b} V_{\text {divfree }}
$$

where $E_{a b}=\sqrt{\bar{g}} \varepsilon_{a b}$ is the 2-dimensional Levi-Civita tensor (having zero projections outside the surfaces of transitivity of the $\mathrm{SO}(3)$ symmetry), with $\varepsilon_{a b}$ the 2dimensional alternating symbol (with the sign convention $\varepsilon_{\theta \varphi}=1$ when polar coordinates are adapted). A similar decomposition holds for any symmetric tensor on spherically symmetric background into scalar, rotationfree and divergencefree parts:

$$
\begin{aligned}
S_{a b}= & S_{b a}=\bar{g}_{a b} S_{\text {scalar }}+\bar{D}_{a} \bar{D}_{b} S_{\text {rotfree }} \\
& +\frac{1}{2}\left(E^{c}{ }_{a} \bar{D}_{c} \bar{D}_{b}+E_{b}^{c} \bar{D}_{c} \bar{D}_{a}\right) S_{\text {divfree }}
\end{aligned}
$$

The scalar and rotationfree parts in the above decompositions compose the even sector under parity transformations, while the divergencefree parts form the odd sector. These sectors decouple. We decompose all metric perturbations as follows

$$
\begin{aligned}
\delta N_{a}= & \bar{D}_{a} P+E^{b}{ }_{a} \bar{D}_{b} Q \\
\delta M_{a}= & \bar{D}_{a} V+E_{a}^{b} \bar{D}_{b} W \\
\delta g_{a b}= & \bar{g}_{a b} A+\bar{D}_{a} \bar{D}_{b} B \\
& +\frac{1}{2}\left(E^{c}{ }_{a} \bar{D}_{c} \bar{D}_{b}+E^{c}{ }_{b} \bar{D}_{c} \bar{D}_{a}\right) C .
\end{aligned}
$$

In consequence the odd sector contains the variables:

$$
Q, W, C
$$

while the even sector is composed of the variables:

$$
P, V, A, B, \delta N, \delta \mathcal{N}, \delta M, \delta \phi
$$

Next we proceed to fix the gauge in an unambiguous manner. For this we need the transformation of the metric and scalar perturbations under diffeomorphisms. The transformed quantities will carry an overhat and they arise as

$$
\mathfrak{L}_{\xi} \tilde{g}_{a b}=\delta \tilde{g}_{a b}-\widehat{\delta \tilde{g}_{a b}}, \quad \mathfrak{L}_{\xi} \phi=\delta \phi-\widehat{\delta \phi}
$$

where the vector $\xi^{a}$ is also decomposed into even and odd contributions

$$
\left(\xi^{t}, \xi^{\chi}, \xi^{a}=\bar{D}^{a} \xi+E^{b a} \bar{D}_{b} \eta\right), \quad(a=\theta, \varphi)
$$

The transformed quantities were given in Ref. [6] as: 


$$
\begin{aligned}
& \widehat{\delta N}=\delta N-\bar{N} \dot{\xi}^{t}-\bar{N}^{\prime} \xi^{\chi}, \\
& \widehat{\delta \mathcal{N}}=\delta \mathcal{N}-\frac{\bar{N}^{2}}{2 \bar{M}^{2}} \xi^{t^{\prime}}+\frac{\bar{M}}{2} \dot{\xi}, \\
& \widehat{\delta M}=\delta M+\bar{M}^{\prime} \xi^{\chi}+\bar{M} \xi^{\chi^{\prime}}, \\
& \widehat{P}=P-\bar{N}^{2} \xi^{t}+\dot{\xi}, \\
& \widehat{Q}=Q+\dot{\eta} \text {, } \\
& \widehat{V}=V+\bar{M}^{2} \xi^{\chi}+\xi^{\prime}-\frac{2}{\chi} \xi, \\
& \widehat{W}=W+\eta^{\prime}-\frac{2}{\chi} \eta, \\
& \widehat{A}=A+\frac{2}{\chi} \xi^{\chi}, \\
& \widehat{B}=B+2 \xi, \\
& \widehat{C}=C+2 \eta, \\
& \widehat{\delta \phi}=\delta \phi-\bar{\phi}^{\prime} \xi^{\chi} \text {. }
\end{aligned}
$$

Here an overdot and a comma denote time derivative and $\chi$-derivative, respectively. It is immediate to consume the radial diffeomorphism d.o.f. for maintaining the radial unitary gauge after the perturbation kicks in, hence $\widehat{\delta \phi}=0$ (e.g. we chose the $\chi$ coordinate on the perturbed spacetime such that constant $\chi$-hypersurfaces and constant $\widehat{\phi}$-hypersurfaces coincide) and

$$
\xi^{\chi}=\frac{\delta \phi}{\bar{\phi}^{\prime}} .
$$

Then we consume the angular diffeomorphisms by rendering the transformation of the 2-metric to a conformal transformation, hence $\widehat{B}=0=\widehat{C}$. This is achieved by the choices

$$
\xi=-\frac{B}{2}, \quad \eta=-\frac{C}{2} .
$$

The last, temporal diffeomorphism was employed in Ref. [6] to reinforce the perpendicularity of the two foliations, hence $\widehat{\delta \mathcal{N}}=0$ and

$$
\xi^{t}=\int d \chi \frac{2 \bar{M}}{\bar{N}^{2}}\left(\delta \mathcal{N}+\frac{\bar{M}}{2} \dot{\xi}^{\chi}\right)+F(t, \theta, \varphi),
$$

which introduced there an arbitrary function depending on all variables, but the radial one. This did not affect the analysis of the odd sector, as the function $F$ emerged only in the even sector variables $\widehat{\delta N}$ and $\widehat{P}$, hampering the physical interpretation of the perturbations. By exploring the freedom of the nonorthogonality of the two foliations however we do not have to chose $\xi^{t}$ in this inconvenient way. Indeed, we could fix $\widehat{P}=0$, achieved by

$$
\xi^{t}=\frac{P+\dot{\xi}}{\bar{N}^{2}}
$$

Therefore the analysis of the even sector perturbations can be carried out unambiguously. In summary, with the gauge choice advanced in this section, the remaining odd sector variables

$$
\widehat{Q}, \widehat{W}
$$

are identical with the ones employed in Ref. [6], while the dynamics of the even sector perturbations is described in terms of the variables

$$
\widehat{V}, \widehat{A}, \widehat{\delta N}, \widehat{\delta \mathcal{N}}, \widehat{\delta M}
$$

Note that another unambiguous gauge fixing for spherically symmetric, static black hole perturbations in Horndeski theories (based on a decomposition into spherical harmonics) has been advanced in Ref. [29], however that choice prefers to cancel the perturbation of the 2-metric rather than preserving the radial unitary gauge. We summarize the various available gauge choices in Table IV. The closest to the Regge-Wheeler gauge is the one developed here, which also ensures the radial unitary gauge.

\section{CONCLUDING REMARKS}

Supplementing existing spacetime decomposition techniques, in this paper we have developed the decomposition along nonorthogonal double foliations. Suppressing the orthogonality requirement of the formalism of Ref. [30], applied successfully in Ref. [6] for the analysis of the odd sector perturbations of beyond-Horndeski theories, but making impossible the similar discussion of the even sector, the latter restriction is lifted. The development of the $2+1+1$ nonorthogonal decomposition formalism followed closely its orthogonal counterpart. The metric has tensorial, vectorial and scalar contributions respective to the intersections of the leaves $\Sigma_{t \chi}$. The 2metric $g_{a b}$, radial shift $M^{a}$ and radial lapse $M$ are canonical coordinates, supplemented by the 2-projection $N^{a}$, radial projection $\mathcal{N}$ of the temporal shift, and the temporal lapse $N$. With two nonorthogonal foliations, there are two different adapted bases. We gave the temporal and radial evolutions in terms of the bases associated with both foliations.

The metric variable $\mathcal{N}$, absent in the orthogonal double foliation formalism, has been found as being related to (i) the Lorentz rotation with angle $\phi=\tanh ^{-1}(\mathcal{N} / N)$, among these bases, and (ii) the vorticity of the basis vectors orthogonal to both the hypersurface normals (of the same basis) and to $\Sigma_{t \chi}$. In the orthogonal limit of vanishing $\mathcal{N}$, the bases coincide and all basis vectors become hypersurface-orthogonal. The 10 th metric variable $\mathcal{N}$ has been reintroduced as a nonorthogonality measure of the formalism.

Then the codimension- 2 embedding of $\Sigma_{t \chi}$ has been characterized in terms of extrinsic curvatures, normal fundamental forms and normal fundamental scalars defined for its normals for both bases and their network of 


\begin{tabular}{|c|c|c|c|c|c|}
\hline & \multicolumn{2}{|c|}{ odd perturbations } & \multicolumn{3}{|c|}{ even perturbations } \\
\hline & vanishing & physical & vanishing & physical & nonvanishing, nonphysical \\
\hline RW & $\widehat{C}=0$ & $\widehat{Q}, \widehat{W}$ & $\widehat{B}=\widehat{P}=\widehat{V}=0$ & $\widehat{\delta N}, \widehat{\delta \mathcal{N}}, \widehat{\delta M}, \widehat{A}$ & \\
\hline KMS & $\widehat{C}=0$ & $\widehat{Q}, \widehat{W}$ & $\widehat{B}=\widehat{P}=\widehat{A}=0$ & $\widehat{\delta N}, \widehat{\delta \mathcal{N}}, \widehat{\delta M}, \widehat{V}, \widehat{\delta \phi}$ & \\
\hline KGT & $\widehat{C}=0$ & $\widehat{Q}, \widehat{W}$ & $\widehat{B}=\widehat{\delta \phi}=0$ & $\widehat{\delta M}, \widehat{A}, \widehat{V}$ & $\widehat{\delta N}, \widehat{\delta \mathcal{N}}, \widehat{P}$ \\
\hline GKG & $\| \widehat{C}=0$ & $\widehat{Q}, \widehat{W}$ & $\widehat{B}=\widehat{P}=\widehat{\delta \phi}=0$ & $\widehat{\delta N}, \widehat{\delta \mathcal{N}}, \widehat{\delta M}, \widehat{A}, \widehat{V}$ & \\
\hline
\end{tabular}

TABLE IV: Comparison of the various gauge choices from the literature for the odd and even sector perturbations (all transcribed in the notations of this paper). In the absence of the scalar field and employing a decomposition into spherical harmonics and their derivatives, Regge and Wheeler (RW) have adopted a unanimous gauge choice leaving 2 odd and 4 even sector metric perturbations [4]. Their approach has been generalized for Horndeski theories by Kobayashi, Motohashi, and Suyama (KMS), resulting in the same 2 variables for the odd sector [28] and 5 for the even sector (these include the scalar field perturbation) [29]. Only 3 out of 4 metric perturbation variables correspond to those of the Regge-Wheeler choice. In the orthogonal double foliation formalism, Kase, Gergely, and Tsujikawa (KGT) have employed the Regge-Wheeler gauge for the odd sector and additionally the radial unitary gauge [6]. The price to pay for the orthogonality of the foliations was an arbitrary function of time appearing in 3 of the even sector metric perturbation variables (nevertheless the even sector was beyond the scope of that paper). In our paper (GKG) we advance another unambiguous gauge choice for scalar-tensor gravity, containing the Regge-Wheeler gauge for the odd sector and all variables corresponding to the even sector analysis of Regge and Wheeler, with an additional one (the even sector part of the metric perturbation $\delta M^{a}$ ). This resulted from imposing the radial unitary gauge, which adapts the $\chi$-coordinate to absorb the scalar field perturbation.

interrelations established. The study of the kinematics of the canonical data indicated that the basis containing the normal to the spatial hypersurfaces is more advantageous, as the embedding variables contain fewer time derivatives. Hence we explored the quantities related to this basis in the remaining part of the paper.

As a first application of the spacetime decomposition along a nonorthogonal double foliation we derived the general relativistic vacuum Hamiltonian dynamics in a fashion similar to Ref. 31], first $2+1+1$ decomposing the curvature scalar in this formalism (and correcting the coefficients in the respective previous result), then giving both the canonical coordinates $g^{A}$ and the canonical momenta $\pi_{A}$ in terms of the introduced geometrical quantities. Their dynamics has been worked out as canonical equations involving the Hamiltonian and diffeomorphism constraints. The expressions derived reproduced all terms emerging in the orthogonal double foliation formalism, supplemented by new terms containing $\mathcal{N}$.

In Appendix $\mathrm{C}$ of Ref. 31] it has been shown that the further $2+1$ decomposition employed in the canonical $3+1$ ADM formalism leads to the $2+1+1$ Hamiltonian formalism with orthogonal double foliation presented there. By relaxing the orthogonality of the foliations, with a similar technique, the Hamiltonian formalism presented here can also be derived. Notably exploring the $2+1$ decompositions of the 3 -dimensional shift

$$
\hat{N}^{a}=N^{a}+\mathcal{N} m^{a}
$$

and of the diffeomorphism constraint

$$
\widehat{\mathcal{H}}_{a}^{G}=\mathcal{H}_{a}^{G}+\mathcal{H}_{\mathcal{N}}^{G} m_{a}
$$

the vacuum GR gravitational Hamiltonian density (54) emerges. Similarly with the $2+1$ decomposition of the induced 3-metric (A1) and of the 3-dimensional canonical momentum

$$
\hat{\pi}^{a b}=\pi^{a b}+M p^{(a} m^{b)}+\frac{M}{2} p m^{a} m^{b}
$$

inserted in the respective equations of the standard ADM approach the $2+1+1$ decomposed action (53)-(58) and Hamiltonian equations of motion (64) and (68)-(70) of this paper follow. Hence the $2+1+1$ decomposition and the variational principle commute.

As compared to the treatment of Ref. [31] a new constraint emerged due to nonorthogonality, the radial diffeomorphism constraint. With this we reestablished the full constraint structure of general relativity, adapted to the nonorthogonal double foliation. In a canonical quantum gravity theory the diffeomorphism constraints must annihilate the physical states. Singling out the radial diffeomorphism constraint through the $2+1$ decomposition of the 3 -dimensional diffeomorphism constraint may turn useful in midisuperspace models [46 48], where integration over the angular sector is carried out and the relevant diffeomorphism constraint is exactly $\mathcal{H}_{\mathcal{N}}^{G}$.

In the last section we proceeded with the second application of our newly developed formalism, the gauge fixing of generic scalar-tensor gravitational theories. As compared to Ref. [6] an unambiguous gauge fixing has been achieved. This includes restricting the perturbation of the 2-metric to a conformal transformation, freezing the evolution of the scalar field by ensuring the radial unitary gauge both before and after the perturbation, and suppressing the even modes of the 2-dimensional shift perturbation. This last step was not possible with the previous assumption of orthogonal double foliation, explored in Ref. [6].

Our work opens up the perspective for discussion of the perturbations of spherically symmetric, static black 
holes in the effective field theory approach of scalartensor gravitational theories in the radial unitary gauge, with the inclusion of both the even and odd sectors.

\section{Acknowledgements}

We are thankful for Zoltán Kovács for help in comparing our results with his previous work. This work was supported by the Hungarian National Research Development and Innovation Office (NKFIH) in the form of the Grant No. 123996 and has been carried out in the framework of COST actions CA15117 (CANTATA) and CA16104 (GWverse), supported by COST (European Cooperation in Science and Technology). C.G. was supported by the UNKP-18-2 New National Excellence Program of the Ministry of Human Capacities of Hungary. Z.K. was supported by the János Bolyai Research Scholarship of the Hungarian Academy of Sciences and by the UNKP-18-4 New National Excellence Program of the Ministry of Human Capacities of Hungary.

\section{Appendix A: Consequences of the hypersurface orthogonality of the basis vectors $\{n, l\}$}

We denote the spatial and Lorentzian 3-metrics induced on the $\mathcal{S}_{t}$ and $\mathfrak{M}_{\chi}$ hypersurfaces, respectively as

$$
\begin{aligned}
& \hat{g}_{a b}=m_{a} m_{b}+g_{a b}, \\
& \check{g}_{a b}=-k_{a} k_{b}+g_{a b} .
\end{aligned}
$$

The basis vectors $n^{a}\left(l^{a}\right)$ being orthogonal to the $\mathcal{S}_{t}\left(\mathfrak{M}_{\chi}\right)$ hypersurfaces, the dual form of the Frobenius theorem guarantees the vanishing of their 3-dimensional vorticities:

$$
\begin{aligned}
& \hat{\omega}_{a b}^{(\mathbf{n})} \equiv \hat{g}_{[a}^{c} \hat{g}_{b]}^{d} \tilde{\nabla}_{c} n_{d}=0, \\
& \check{\omega}_{a b}^{(\mathbf{l})} \equiv \check{g}_{[a}^{c} \check{g}_{b]}^{d} \tilde{\nabla}_{c} l_{d}=0 .
\end{aligned}
$$

These can be further $2+1$ decomposed as

$$
\begin{aligned}
& 0=D_{[a} n_{b]}+m_{[a} g_{b]}^{d} m^{c}\left(\tilde{\nabla}_{c} n_{d}-\tilde{\nabla}_{d} n_{c}\right), \\
& 0=D_{[a} l_{b]}+k^{c} g_{[a}^{d} k_{b]}\left(\tilde{\nabla}_{c} l_{d}-\tilde{\nabla}_{d} l_{c}\right),
\end{aligned}
$$

the contraction of which with $m^{a}$ and $k^{a}$, respectively leading to the second type of expressions of the normal fundamental forms, given in Eqs. (14).

Projecting Eqs. (A5) and (A6) to $\Sigma_{t \chi}$ confirms the vanishing of the 2 -dimensional vorticities:

$$
\begin{gathered}
\omega_{a b}^{(\mathbf{n})} \equiv D_{[a} n_{b]}=0, \\
\omega_{a b}^{(\mathbf{l})} \equiv D_{[a} l_{b]}=0,
\end{gathered}
$$

and symmetry of the extrinsic curvatures

$$
\begin{aligned}
K_{a b} & \equiv D_{a} n_{b}=D_{(a} n_{b)}=K_{b a} \\
L_{a b} & \equiv D_{a} l_{b}=D_{(a} l_{b)}=L_{b a},
\end{aligned}
$$

which could also be directly checked from the first Eq. (7) and the second Eq. (12), giving the normals

$$
\begin{aligned}
n_{a} & =-N \tilde{\nabla}_{a} t, \\
l_{a} & =M \mathfrak{c} \tilde{\nabla}_{a} \chi .
\end{aligned}
$$

Inserting these into the definitions manifestly symmetric expressions arise.

\section{Appendix B: Consequences of the vorticity of the basis vectors $\{k, m\}$}

We introduce 3-dimensional metrics, which are orthogonal to the basis vectors $k^{a}$ and $m^{a}$, respectively:

$$
\begin{aligned}
& \hat{h}_{a b}=l_{a} l_{b}+g_{a b}, \\
& \check{h}_{a b}=-n_{a} n_{b}+g_{a b} .
\end{aligned}
$$

These metrics are defined on 3-manifolds which are not hypersurfaces, but rather the manifolds formed by the integral curves of the vector fields $k^{a}$ and $m^{a}$. The 3dimensional vorticity tensors of $k^{a}$ and $m^{a}$ do not vanish, as they are not hypersurface-orthogonal:

$$
\begin{gathered}
\hat{\omega}_{a b}^{(\mathbf{k})} \equiv \hat{h}_{[a}^{c} \hat{h}_{b]}^{d} \tilde{\nabla}_{c} k_{d} \neq 0, \\
\check{\omega}_{a b}^{(\mathbf{m})} \equiv \check{h}_{[a}^{c} \check{h}_{b]}^{d} \tilde{\nabla}_{c} m_{d} \neq 0 .
\end{gathered}
$$

Their $2+1$ decomposition leads to

$$
\begin{aligned}
& \hat{\omega}_{a b}^{(\mathbf{k})}=g_{[a}^{c} g_{b]}^{d} \tilde{\nabla}_{c} k_{d}+l_{[a} g_{b]}^{d} l^{c}\left(\tilde{\nabla}_{c} k_{d}-\tilde{\nabla}_{d} k_{c}\right), \\
& \check{\omega}_{a b}^{(\mathbf{m})}=g_{[a}^{c} g_{b]}^{d} \tilde{\nabla}_{c} m_{d}+g_{[a}^{d} n_{b]} n^{c}\left(\tilde{\nabla}_{c} m_{d}-\tilde{\nabla}_{d} m_{c}\right)
\end{aligned}
$$

Projecting Eqs. (B5) and (B6) to the $\Sigma_{t \chi}$ surfaces leads to the 2-dimensional vorticities:

$$
\begin{gathered}
\omega_{a b}^{(\mathbf{k})} \equiv D_{[a} k_{b]}=0, \\
\omega_{a b}^{(\mathbf{m})} \equiv D_{[a} m_{b]}=0,
\end{gathered}
$$

which (as both $k^{a}$ and $m^{a}$ are orthogonal to the surface $\left.\Sigma_{t \chi}\right)$ vanish due to the dual form of the Frobenius theorem. Alternatively, these can be proved directly through the relations

$$
\begin{aligned}
k_{a} & =M \mathfrak{s} \tilde{\nabla}_{a} \chi-\frac{N}{\mathfrak{c}} \tilde{\nabla}_{a} t, \\
m_{a} & =N \frac{\mathfrak{s}}{\mathfrak{c}} \tilde{\nabla}_{a} t+M \tilde{\nabla}_{a} \chi,
\end{aligned}
$$

emerging from the second Eq. (7) and the first Eq. (12). With them the symmetry of the extrinsic curvatures $K_{a b}^{*}$ and $L_{a b}^{*}$ can be readily checked. 


\begin{tabular}{|l|l|}
\hline$\hat{\omega}_{a b}^{(\mathbf{k})} g_{c}^{b}=0$ & $\check{\omega}_{a b}^{(\mathbf{m})} g_{c}^{b}=0$ \\
\hline$\hat{\omega}_{a b}^{(\mathbf{k})} l^{b}=\frac{1}{2} D_{a} \phi-\frac{\mathfrak{s}}{2 c}\left(\mathfrak{a}_{a}+\mathfrak{b}_{a}\right)$ & $\check{\omega}_{a b}^{(\mathbf{m})} n^{b}=\frac{1}{2} D_{a} \phi+\frac{\mathfrak{I}}{2 \mathfrak{c}}\left(\mathfrak{a}_{a}+\mathfrak{b}_{a}\right)$ \\
\hline
\end{tabular}

TABLE V: The 3-dimensional vorticity components in terms of $\phi($ or $\mathcal{N})$.

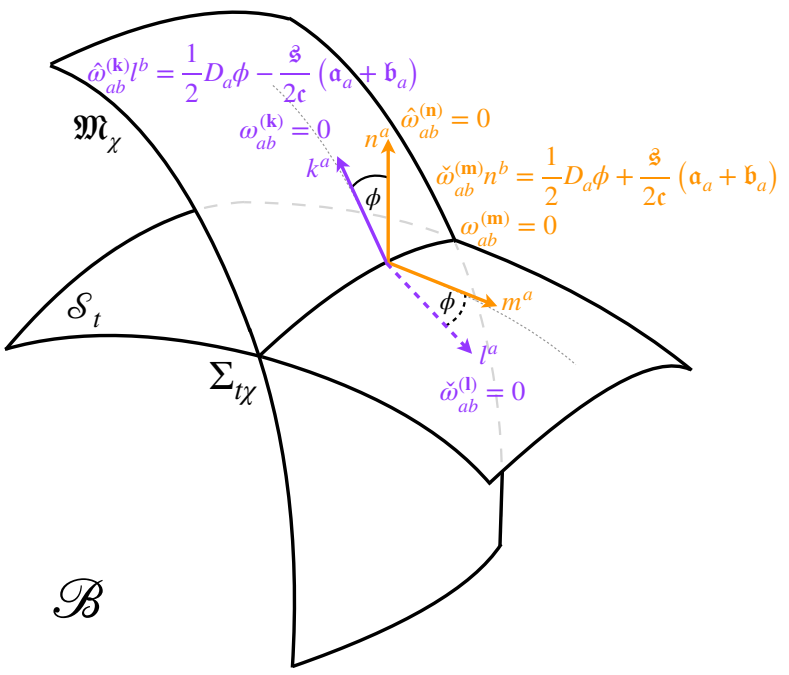

FIG. 4: The nonvanishing vorticity components of the basis vectors.

Hence the 3-dimensional vorticities reduce to:

$$
\begin{gathered}
\hat{\omega}_{a b}^{(\mathbf{k})}=l_{[a} g_{b]}^{d} l^{c}\left(\tilde{\nabla}_{c} k_{d}-\tilde{\nabla}_{d} k_{c}\right), \\
\check{\omega}_{a b}^{(\mathbf{m})}=-n_{[a} g_{b]}^{d} n^{c}\left(\tilde{\nabla}_{c} m_{d}-\tilde{\nabla}_{d} m_{c}\right),
\end{gathered}
$$

having nonvanishing components only along the normals of the two hypersurface families. These nonvanishing vorticity components of the basis vectors are also indicated on Fig. 4 .

By exploring the definitions (14) and (16) we get the expressions of the starry quantities $\mathcal{K}_{a}^{*}$ and $\mathcal{L}_{a}^{*}$ in terms of normal fundamental forms and nonvanishing 3dimensional vorticity components:

$$
\begin{aligned}
\mathcal{K}_{a}^{*} & =\mathcal{L}_{a}-2 \hat{\omega}_{a b}^{(\mathbf{k})} l^{b}, \\
\mathcal{L}_{a}^{*} & =\mathcal{K}_{a}+2 \check{\omega}_{a b}^{(\mathbf{m})} n^{b} .
\end{aligned}
$$

By exploring the results given in Table III, by straightforward algebra we can express the 3 -dimensional vorticities in terms of the 10th metric variable, as given in Table V
[1] G.W. Horndeski, Second-order scalar-tensor field equations in a four-dimensional space, Int. J. Theor. Phys. 10, 363 (1974).

[2] C. Deffayet, X. Gao, D. A. Steer, and G. Zahariade, From k-essence to generalized Galileons, Phys. Rev. D 84, 064039 (2011).

[3] J. Gleyzes, D. Langlois, F. Piazza, and F. Vernizzi, Healthy theories beyond Horndeski, Phys. Rev. Lett. 114, 211101 (2015) arXiv:1404.6495 [hep-th]].

[4] J. Gleyzes, D. Langlois, F. Piazza, and F. Vernizzi, Essential building blocks of dark energy, J. Cosmol. Astropart. Phys. 08 (2013) 025 arXiv:1304.4840 [hep-th]].

[5] L. Á. Gergely and S. Tsujikawa, Effective field theory of modified gravity with two scalar fields: Dark energy and dark matter, Phys. Rev. D 89, 064059 (2014) arXiv:1402.0553 [hep-th]].

[6] R. Kase, L. Á. Gergely, and S. Tsujikawa, Effective field theory of modified gravity on spherically symmetric background: leading order dynamics and the odd mode perturbations, Phys. Rev. D 90, 124019 (2014) arXiv:1406.2402 [hep-th]].

[7] R. Kimura, T. Kobayashi, and K. Yamamoto, Vainshtein screening in a cosmological background in the most general second-order scalar-tensor theory, Phys. Rev. D 85, 024023 (2012) arXiv:1111.6749 [astro-ph.CO]].

[8] R. Kase and S. Tsujikawa, Screening the fifth force in the Horndeski's most general scalar-tensor theories, J. Cosmol. Astropart. Phys. 08 (2013) 054 arXiv:1306.6401 [gr-qc]].

[9] K. Koyama, G. Niz, and G. Tasinato, Effective theory for the Vainshtein mechanism from the Horndeski action, Phys. Rev. D 88, 021502(R) (2013) arXiv:1305.0279 [hep-th]].

[10] J. Sakstein, H. Wilcox, D. Bacon, K. Koyama, and R. C. Nichol, Testing Gravity Using Galaxy Clusters: New Constraints on Beyond Horndeski Theories, J. Cosmol. Astropart. Phys. 07 (2016) 019 arXiv:1603.06368 [astroph.CO]].

[11] LIGO Scientific and Virgo Collaborations, Observation of Gravitational Waves from a Binary Black Hole Merger, Phys. Rev. Lett. 116, 061102 (2016) arXiv:1602.03837 [gr-qc]].

[12] LIGO Scientific and Virgo Collaborations, GW151226: Observation of Gravitational Waves from a 22-SolarMass Binary Black Hole Coalescence, Phys. Rev. Lett. 
116, 241103 (2016) arXiv:1606.04855 [gr-qc]].

[13] LIGO Scientific and Virgo Collaborations, GW170104: Observation of a 50-Solar-Mass Binary Black Hole Coalescence at Redshift 0.2, Phys. Rev. Lett. 118, 221101 (2017) arXiv:1706.01812 [gr-qc]].

[14] LIGO Scientific and Virgo Collaborations, GW170608: Observation of a 19-Solar-Mass Binary Black Hole Coalescence, Astrophys. J. Lett. 851, L35 (2017) arXiv:1711.05578 [astro-ph.HE]].

[15] LIGO Scientific and Virgo Collaborations, GW170814: A Three-Detector Observation of Gravitational Waves from a Binary Black Hole Coalescence, Phys. Rev. Lett. 119, 141101 (2017) arXiv:1709.09660 [gr-qc]].

[16] LIGO Scientific and Virgo Collaborations, GW170817: Observation of Gravitational Waves from a Binary Neutron Star Inspiral, Phys. Rev. Lett. 119, 161101 (2017) arXiv:1710.05832 [gr-qc]].

[17] LIGO Scientific and Virgo Collaborations, GWTC1: A Gravitational-Wave Transient Catalog of Compact Binary Mergers Observed by LIGO and Virgo during the First and Second Observing Runs, (2018) [arXiv:181112907 [astro-ph.HE]].

[18] LIGO Scientific and Virgo Collaborations, Tests of General Relativity with GW150914, Phys. Rev. Lett. 116, 221101 (2016) arXiv:1602.03841 [gr-qc]].

[19] S. Mirshekari, N. Yunes, and C. M. Will, Constraining Lorentz-violating, Modified Dispersion Relations with Gravitational Waves, Phys. Rev. D 85, 024041 (2012) arXiv:1110.2720 [gr-qc]].

[20] LIGO Scientific and Virgo Collaborations, Tests of General Relativity with the Binary Black Hole Signals from the LIGO-Virgo Catalog GWTC-1, (2019) arXiv:1602.03837 [gr-qc]].

[21] LIGO Scientific and Virgo Collaborations, Fermi Gamma-ray burst monitor, and INTEGRAL, Gravitational Waves and Gamma-Rays from a Binary Neutron Star Merger: GW170817 and GRB170817A, Astrophys. J. Lett. 848, L13 (2017).

[22] T. Kobayashi, M. Yamaguchi, and J. Yokoyama, Generalized G-inflation: Inflation with the most general secondorder field equations, Prog. Theor. Phys. 126, 511-529 (2011) arXiv:1105.5723 [hep-th]].

[23] A. De Felice and S. Tsujikawa, Conditions for the cosmological viability of the most general scalar-tensor theories and their applications to extended Galileon dark energy models, J. Cosmol. Astrophys. Phys. 1202, 007 (2012) arXiv:1110.3878 [gr-qc]].

[24] T. Baker, E. Bellini, P. G. Ferreira, M. Lagos, J. Noller, and I. Sawicki, Strong constraints on cosmological gravity from GW170817 and GRB 170817A, Phys. Rev. Lett. 119, 251301 (2017) arXiv:1710.06394 [astro-ph.CO]].

[25] J. M. Ezquiaga and M. Zumalacárregui, Dark Energy after GW170817: Dead ends and the road ahead, Phys. Rev. Lett. 119, 251304 (2017) arXiv:1710.05901 [astroph.CO]].

[26] P. Creminelli and F. Vernizzi, Dark Energy after GW170817 and GRB170817A, Phys. Rev. Lett. 119, 251302 (2017) arXiv:1710.05877 [astro-ph.CO]].

[27] J. Sakstein and B. Jain, Implications of the Neutron Star Merger GW170817 for Cosmological ScalarTensor Theories, Phys.Rev.Lett. 119, 251303 (2017) arXiv:1710.05893 [astro-ph.CO]].

[28] T. Kobayashi, H. Motohashi and T. Suyama, Black hole perturbation in the most general scalar-tensor theory with second-order field equations I: The odd-parity sector, Phys. Rev. D 85, 084025 (2012) arXiv:1202.4893 [gr-qc]].

[29] T. Kobayashi, H. Motohashi and T. Suyama, Black hole perturbation in the most general scalar-tensor theory with second-order field equations II: the even-parity sector, Phys. Rev. D 89, 084042 (2014) arXiv:1402.6740 [gr-qc]].

[30] L. Á. Gergely and Z. Kovács, Gravitational dynamics in s+1+1 dimensions, Phys. Rev. D 72, 064015 (2005) arXiv:gr-qc/0507020.

[31] Z. Kovács and L. Á. Gergely, Gravitational dynamics in s+1+1 dimensions II. Hamiltonian theory, Phys. Rev. D 77, 024003 (2008) arXiv:0709.2131 [gr-qc]].

[32] S. Chandrasekhar, "The Mathematical Theory of Black Holes", (Oxford Clarendon, 1983).

[33] C. A. Clarkson and R. K. Barrett, Covariant Perturbations of Schwarzschild Black Holes, Class. Quant. Grav. 20, 3855 (2003) gr-qc/0209051.

[34] C. Clarkson, A covariant approach for perturbations of rotationally symmetric spacetimes, Phys. Rev. D 76, 104034 (2007) arXiv:0708.1398 [gr-qc]].

[35] K. Maeda, M. Sasaki, T. Nakamura, and S. Miyama, A New Formalism of the Einstein Equations for Relativistic Rotating Systems, Prog. Theor. Phys. 63, 719 (1980).

[36] M. Sasaki, [(2+1)+1]-Formalism of General Relativity in Problems of Collapse and Numerical Relativity, edited by D. Bancel and M. Signore (Reidel, Dordrecht, 1984), pp. 203-220.

[37] E. Gourgoulhon and S. Bonazzola, Noncircular axisymmetric stationary spacetimes, Phys. Rev. D 48, 2635 (1993).

[38] E. Gourgoulhon, Generalized Damour-Navier-Stokes equation applied to trapping horizons, Phys. Rev. D 72, 104007 (2005) arXiv:gr-qc/0508003.

[39] E. Gourgoulhon and J. L. Jaramillo, A 3+1 perspective on null hypersurfaces and isolated horizons, Phys. Rep. 423, 159-294 (2006) arXiv:gr-qc/0503113.

[40] I. Rácz, Constraints as evolutionary systems, Class. Quantum Grav. 33015014 (2016) arXiv:1508.01810 [grqc]].

[41] J. A. Schouten, Ricci-Calculus (Springer Verlag, Berlin, 1954).

[42] R. A. d'Inverno and J. Stachel, Conformal two-structure as the gravitational degrees of freedom in general relativity, J. Math. Phys. (N.Y.) 19, 2447 (1978).

[43] R. A. d'Inverno and J. Smallwood, Covariant $2+2$ formulation of the initial-value problem in general relativity, Phys. Rev. D 22, 1233 (1980).

[44] T. Regge and J. A. Wheeler, Stability of a Schwarzschild Singularity, Phys. Rev. 108, 1063 (1957).

[45] F. Zerilli, Perturbation analysis for gravitational and electromagnetic radiation in a Reissner-Nordström geometry, Phys. Rev. D 9, 860 (1974).

[46] K. Kuchař, Canonical Quantization of Cylindrical Gravitational Waves, Phys. Rev. D 4, 955 (1971).

[47] C. G. Torre, Midi-superspace Models of Canonical Quantum Gravity, J. Theor. Phys. 38, 1081 (1999) arXiv:gr-qc/9806122.

[48] M. Bojowald, Canonical Gravity and Applications: Cosmology, Black Holes, and Quantum Gravity (Cambridge, England, 2011). 